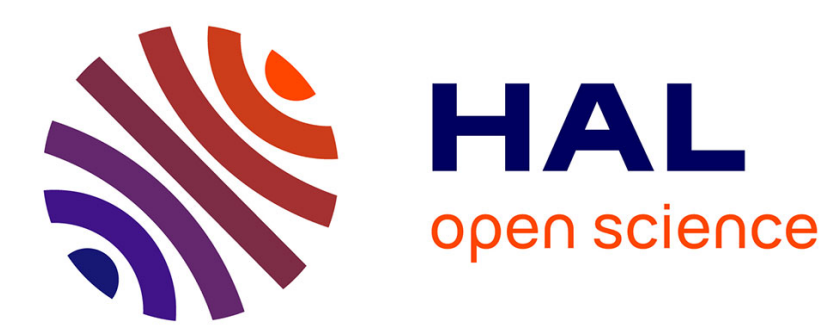

\title{
Nuclear magnetic resonance in ferromagnets: Ferromagnetic nuclear resonance; a very broadband approach
}

Christian Meny, Pierre Panissod

\section{To cite this version:}

Christian Meny, Pierre Panissod. Nuclear magnetic resonance in ferromagnets: Ferromagnetic nuclear resonance; a very broadband approach. Annual reports on NMR spectroscopy, pp.47-96, 2021, 10.1016/bs.arnmr.2021.02.001 . hal-03301643

\section{HAL Id: hal-03301643 \\ https://hal.science/hal-03301643}

Submitted on 27 Jul 2021

HAL is a multi-disciplinary open access archive for the deposit and dissemination of scientific research documents, whether they are published or not. The documents may come from teaching and research institutions in France or abroad, or from public or private research centers.
L'archive ouverte pluridisciplinaire HAL, est destinée au dépôt et à la diffusion de documents scientifiques de niveau recherche, publiés ou non, émanant des établissements d'enseignement et de recherche français ou étrangers, des laboratoires publics ou privés. 
Annual Reports on NMR Spectroscopy

Nuclear magnetic resonance in ferromagnets: ferromagnetic nuclear resonance;

a very broadband approach.

C. Meny* and P. Panissod

Institut de Physique et Chimie des Matériaux de Strasbourg, Université de Strasbourg, CNRS UMR 7504, 23 rue de Loess, BP 43, F-67034 Strasbourg Cedex 2, France

*e-mail : Christian.Meny@ipcms.unistra.fr

Keywords: solid state nuclear magnetic reson 210 films; nanoparticles; interface; structure. 


\section{Abstract}

Nuclear Magnetic Resonance is not often used for studying ferromagnetic materials. Because of the technical particularities resulting from the ferromagnetic nature of the samples it is often called ferromagnetic nuclear resonance (FNR). Its first particularity is that, without applying any external magnetic field, the nuclei already experience a magnetic field originating from the spontaneous magnetisation of the samples. Therefore, FNR spectra have to be recorded by frequency scanning. The second particularity is that the radiofrequency field experienced by the nuclei is not the RF field applied to the sample but is a field enhanced by the sample's electronic magnetic susceptibility. The first consequence is that the optimum FNR excitation power required to measure the spectra is not known and has to be determined for each sample. The second consequence is that the recorded spectrum tor agiven RF field power will not reflect the true nuclei frequency density, making difficut quantitative analyses. In this paper we present the experimental approach we have eveloped to overcome the difficulties resulting from the particularities of FNR. It is presented roth from a conceptual point of view as from an experimental and technical point of ew Examples of recent experimental results where this approach has been particularly efficient are given. Finally, the paper is concluded with the proposition of a design for an FNR spectrometer with automated frequency scanning built from on the shelf electronic components. It will allow to make FNR set ups available to a much broader scientific community. 
1. Introduction

2. Advantage and drawbacks of a very broadband FNR approach

3. Particularities of FNR

3.1. Internal Field

3.2. Enhancement factor

3.3. Distribution of enhancement

3.4. FNR signal versus external RF field amplitude

3.5. Restoring field

4. Computation of the nuclei frequency densit:

4.1. Log Normal model

4.2. Determination of the restoring field

4.3. Role of the high-pass filter probe design in the determination of the restoring field.

4.4. Establishing the true Nuclei frequency density $N(\omega)$

5. Technical aspects

5.1. Double heterodyne FNR spectrometer design

5.2. FRN broadband high pass filter design probe head 


\subsection{Operating mode}

\subsubsection{Pulse sequence}

5.3.2. Fast scanning mode

5.3.3. Performances in the fast scanning mode

\section{Experimental examples}

6.1. Co/organic thin films and investigation of single buried interfaces

6.2. Temperature Differential Ferromagnetic Nuclear Resonance Spectra (TDFNR)

6.3. Magnetic dipolar interactions in assemt,iies ay nano-objects

7. A broadband FNR spectrometer with autrmaied frequency scanning built from on the shelf components

8. Conclusion 


\section{Introduction}

Nuclear magnetic resonance (NMR) is widely used in chemistry biology or medicine. Its use for studying magnetically ordered materials (i.e. ferromagnetic materials) is much scarcer. This is mainly due to the two main particularities of NMR when performed in ferromagnets. The first one is that even without applying any external magnetic field the nuclei already experience a magnetic field that has for origin the spontaneous magnetisation of the samples. Its main contribution, the hyperfine field, has a magnitude of several tens of tesla. Sometime an external magnetic field may be applied in order to identify the observed nucleus (by its gyromagnetic ratio) or to determine the sign of the hyperfine field or to get information on anisotropic hyperfine properties (magnetic hyperfine field tensor or electric quadrupole interaction), or, more seldom, to get rid of unwanted complications arising irom magnetic domain wall effects on the NMR signal. However, except for few sysem inke pure cubic ferromagnetic elements, the distribution of hyperfine fields is in-hom, gen ously spread over a broad frequency range (10-100 MHz typical). This means that tondard field swept technique at fixed frequency used for nonmagnetic materials is ha dy a hievable (not to talk of FFT techniques). Therefore, NMR spectra in ferrimagnets are recorded by frequency scanning. The second particularity of NMR in ferromagnets is that the radiofrequency $(\mathrm{RF})$ field experienced by the nuclei is not the RF field applied to the sample $(H 1)$ but is a field $\left(H I^{*}\right)$ enhanced by the electronic magnetic susceptibility of the sample. The first consequence of this enhancement is that the optimum NMR excitation power required to measure the spectra is not known and has to be determined for each sample. The second consequence results from the fact that since the magnetic susceptibility might differ from one nucleus site to another within the same sample, the recorded spectrum for a specific set of recording conditions (i.e. one specific $H 1$ power) might not reflect the true nuclei frequency density, making difficult a quantitative analysis of the data. Because of all these particularities NMR in ferromagnets is often referred with specific names: 
ferromagnetic nuclear resonance (FNR the one we favour), Zero field NMR or Internal field NMR (IF-NMR).

To overcome the difficulties resulting from the particularities of NMR in ferromagnets (that will be called FNR in the following text) we have developed a very broadband approach. This approach is based on the use of un-tuned FNR spectrometers with automated frequency scanning. It allows to map the FNR signal for a wide range of $H 1$ power. From the mapping of the $H 1$ power FNR signal it is possible to determine the site dependent enhancement factor of the RF field $\mathrm{Hl}$ and correct the FNR response in order to establish FNR spectra that reproduce the true nuclei frequency density. In addition, the determination of the site dependent RF field enhancement factor (rewritten in a form similar to an effective magnetic anisotropy field) gives a unique insight into the local magnetic properties inside tus studied samples.

During the last 3 decades this broadband approa $h$ has been used to study a large variety of samples [1-3], bulk samples [4-10], magnetin nutilayers and thin films [1-51], assemblies of nano-objects [52-62]. It allowed to give ontributions into various research fields: spintronics, hard magnets, multi-layered mirrors, hei- ogeneous catalysis etc.

In this article we will describe our broadband approach and discuss its advantages and drawbacks. For this purpose, we will first remind the origin of the internal magnetic field experienced by the nuclei and explain the RF field enhancement process. It will be followed by the determination of the shape of the FNR response versus the amplitude of the RF field. From the shape of the FNR response an experimental methodology has been developed to establish FNR spectra representing truly the nuclear spins' frequency density. This has been described in early $\mathrm{PhD}$ works $[63,64]$ but has never been published elsewhere. The insight obtained by this methodology into the local magnetic properties of the samples will also be discussed. The second part of the article will focus on the experimental set up and describe the concept of the 
spectrometers that have been used to perform the measurements. We shall focus on the characteristics of the spectrometers that are desirable for a fast, sensitive, reliable and automatic acquisition of very broad FNR spectra. We will especially emphasize about the FNR broadband probe design that is a key component to perform automated frequency scans. Afterward examples of recent experimental results where this approach has been particularly efficient will be described. Finally, the paper will be concluded with the proposition of a design for a FNR spectrometer based from on the shelf electronic components. It will allow to make FNR set ups available to a much broader scientific community. While this new spectrometer is not fully developed yet, proof of concept has already been performed.

\section{Advantage and drawbacks of a very broadbcinc wopisach}

As mentioned in the introduction, FNR spect a have to be recorded by frequency scanning. If using a spectrometer with a tuned probs, th is fact has the consequence to lengthen the

measurement time because of the $n$ essity to tune the probe coil and modify several other settings for each frequency. Another consequence is the necessity to calibrate the instrument amplitude response (both of the receiver and of the transmitter) for each frequency and for each measurement because of unavoidable inaccuracy and irreproducibility of the manual settings. At last such procedure cannot be easily automated and computerized in order to use standard data accumulation techniques for noise and drift reduction. By data accumulation we mean accumulation of spectra and not data accumulation at spot frequencies: both allow short term noise reduction but the latter is subject to all long term drifts and fluctuations.

The first point one faces when designing a broadband spectrometer with automatic frequency scanning is the necessity of using an un-tuned probe. This could be a simple coil (without tuning capacitor) fed at constant voltage or constant intensity by the transmitter. However, owing to 
the existence of transmitting lines of some length from transceiver to probe, owing to the unpredictable response of the transmitter loaded by an inductive network, and for the sake of efficiency, an impedance matching (transceiver to line and line to probe) is wanted. Several designs of broadband matched NMR probes have been proposed in the past which are based on a delay line scheme [65]. As opposed to the low-pass filter design of delay lines, the design we use is based on a high-pass filter design. In any instance such design, once correctly tuned and matched, can provide flat frequency response of either the intensity flowing through the sample coil or the voltage drop across the coil. A side advantage of the flat frequency response of an un-tuned probe is its much better reproducibility between different scans. However, for the same RMS noise output, a tuned probe of quality factor $Q$ yields a signal amplitude about $\sqrt{ } Q$ times larger than an un-tuned coil.

Even if the sensitivity of the spectrometer is redtete when using an un-tuned probe, there are several advantages for using a design with antom ted frequency scanning. Resonances can be sought for much more quickly and more dweniently. Indeed, in ferromagnetic materials the internal hyperfine field is large an un'nown, a priori. Therefore, unlike in nonmagnetic materials, where the NMR frequency is determined mostly by the external field, the resonance frequency of a nucleus in a ferromagnet may be found anywhere in a frequency range covering several hundred MHz.

Thorough studies of the effect of RF power on the whole spectrum shape can be performed very easily. In ferromagnets the nuclei are not directly excited by the external $H I$ but by hyperfine field oscillations which follow the response of the electronic magnetic moments to $H 1$. Reciprocally, the induced voltage in the receiver coil is not directly the nuclear induction but an induction resulting from the electronic moments driven by the nuclear precession. Consequently, as discovered by Portis and Gossard [66], the effective RF field experienced by 
the nuclei, $H l^{*}$ and the FNR signal are largely enhanced by a factor $\eta$ with typical values ranging from $10^{2}$ to $10^{4}$. If one needs accurate relative (and ultimately absolute) intensities, the measurement of $\eta$ for each frequency is necessary in order to correct for any possible frequency dependence. The knowledge of $\eta$ for the various lines of a spectrum is also needed in order to assign the respective signals to different kinds of electronic response to $H l$ (magnetic domain rotation or magnetic domain wall motion) [6] which gives a useful insight on the local magnetic properties of a sample. Moreover, since $\eta$ is related to the local magnetic stiffness (permeability) of the electronic moments, it differs from place to place in an inhomogeneous or composite sample. Therefore, the variation of $\eta$ along the spectrum allows to discriminate between the various phases, the various atomic and magnetic configurations which are present in many complex modern materials. A convenient wav to measure $\eta$ is to record NMR spectra for different strengths of $H I$ covering a range ovf nion than one order of magnitude. The raw data result in a 3D picture (Signal intensity a a ilunction of both frequency and $H 1$ strength). This will be explained in detail further in this sticle.

In similar ways, studies of the relaxatıcines $T_{1}$ and $T_{2}$ along the spectrum may also serve the purpose of discriminating between various local environments or phases as well as the investigation of the dynamic magnetic properties.

Such studies, which are unusual kinds of 2D FNR, imply recording many spectra for the same sample (with different $H 1$ strengths and/or delays) and are quite time consuming. This is much easier to undertake if frequency scanning is automated

And finally if sensitivity is still a concern, the fact that the recording of the spectra is fully automated, makes possible to perform measurements during much longer times, allowing, at least partially, to compensate for the reduced sensitivity. 


\section{Particularities of FNR}

\subsection{Internal Field}

The first particularity resulting from the study of ferromagnetic systems is that even without applying any external magnetic field the nuclei already experience a magnetic field, $\mathrm{Hm}$, that has for origin the spontaneous magnetisation of the samples [67]. The total static field $\mathrm{HO}$ experienced by the nuclei can be expressed as the vector sum:

$$
\begin{aligned}
& H O=H e x t+H m \\
& H O=H e x t+H d i p+H F
\end{aligned}
$$

Where

Hext is an external field (not required in Five).

$\mathrm{Hm}$ is the field resulting from the spontaneous magnetization of the sample.

$H F$ is the hyperfine field due to electron spin and orbital moments within the ion radius.

Hdip is the dipolar field resulting from other moments in the sample.

The classical dipolar field is split into three components:

$$
\text { Hdip }=\text { Hdem }+ \text { HLor }+ \text { HLoc }
$$

where

Hdem is the demagnetizing field related to the macroscopic shape of the sample.

HLor is the Lorentz cavity field. 
HLoc is the field due to all moments in the Lorentz sphere except the central one.

It can be noted that $H d e m+H L o r=0$ for a spherical sample and $H L o c=0$ for a cubic site symmetry.

The hyperfine field HF is the sum of three contributions within the ion radius:

$$
H F=H F c o n+H F d i p+H F o r b
$$

Where

HFdip is the dipolar field from electron spins outside of the nucleus volume.

HForb is the orbital field, associated with a not fully quenched orbital moment.

Hcon is the Fermi contact field which orisinates from the spin polarization of all electrons (essentially s shells) within the nucleus voivme.

It is usual to group HLoc, HFdip , and th criscropic part of HForb into what is called the anisotropic hyperfine field HFani (stricti's speaking, a traceless second rank tensor).

The contact field HFcon is isotropic, as being a spin contribution. For $3 \mathrm{~d}$ ferromagnetic materials, it is the largest contribution to the hyperfine field (for Co, HFcon $20 \mathrm{~T}$ ).

Three contributions to the spin polarization at the nucleus can be distinguished:

$$
\text { HFcon }=\text { HFcore }+ \text { HFcond }+ \text { HFtran }
$$

where

HFcore arises from the core polarization due to the exchange interaction between "s" electrons of the inner shells and the on-site magnetic moment of the d electrons, it is generally negative, i.e., antiparallel to the local moment. 
HFcond arises from the spin polarization of the "s" conduction electrons due to the onsite magnetic moment of the atom itself.

HFtran arises from the conduction electron polarization due to the moments of neighbouring atoms (transferred hyperfine field).

For paramagnetic and diamagnetic materials, the NMR resonance frequency is mainly determined by the externally applied magnetic field $\mathrm{HO}$ and the magnetic hyperfine interaction results only in a small frequency shift due to the weak polarization of unpaired electron spins, if any, or the very small perturbation of the orbital motion of the filled shells by the external field (Knight and chemical shifts). In ferromagnetic materials, owing to the spontaneous magnetization, the $H F$ is much larger (20-30 $\mathrm{T}$ for the ${ }^{2} \mathrm{~d}$ elements) and becomes the dominant factor inducing the splitting of the nuclear energy veis (and therefore the resonance can be observed without an externally applied magnetic tield).

\subsection{Enhancement factor}

As described above in FNR the use of an external DC field is not necessary because the spontaneous magnetic moment creates a large internal field, at the nuclei. The second particularity when performing NMR in ferromagnets is that the nuclei are not directly excited by the external radio-frequency field $H 1$ but by the oscillation of the $H F$ following the electronic moment response to $H 1$. This field is usually called $H l^{*}$. The enhancement factor $\eta$ is defined by:

$\eta=H 1 * / H 1$. 
The position at rest of the electronic moment is determined by its minimum energy (magnetic anisotropy, dipolar and exchange energy, etc.).

As far as the received signal is concerned, the motion of the transverse nuclear magnetisation exerts a torque on the electronic magnetisation which is thus driven into rotation (or oscillation). Thus the electronic magnetisation acquires an oscillating component $\eta$ times larger than the rotating nuclear component. Since the actual signal in the probe coil is mostly induced by the motion of the electronic magnetisation it is thus enhanced by the same factor $\eta$ as the RF field excitation. As a consequence, in ferromagnets, the FNR signal should read:

$$
s\left(\omega, H_{1}\right)=K \eta \omega \chi_{n} H F R\left[\theta\left(\eta H_{1}\right)\right]
$$

where

$\chi_{n}$ is the nuclear susceptibility and $\chi_{\mathrm{n}} \mathrm{HF}$ is herefore the nuclear magnetisation.

$\omega$ arises from the fact that the sigval alts from the variation of a magnetic flux in the probe coil.

$\theta$ is the turn angle of the nuclear magnetisation resulting from the pulse sequence.

$K$ is a constant experimental factor.

Therefore, equation 7 can be written

$$
s\left(\omega, H_{1}\right)=K \eta R[\theta(\eta H 1)] \omega^{2} N(\omega) \not(I+1) / T
$$

where

$N(\omega)$ is the nuclei frequency density: the "true" FMR intensity.

$R$ is the nuclear spin response. 
$\gamma$ is the nucleus gyro magnetic ratio.

$I$ is the nucleus spin

$T$ is the measurement temperature.

\subsection{Distribution of enhancement}

In ferromagnetic materials the enhancement factor will usually vary from place to place therefore there will be a distribution of enhancement inside the material. Therefore, a more complete description than above is needed. When the external RF field $H 1$ is applied, the resulting rotation $d \theta$ of the electronic magnetisation indus a rotation $d \phi$ of the $H F$ which produces a field component $H I^{*}=H F d \phi$ perpentictiar to $H F$ (see Figure 1 ). The enhancement factor is thus written as

$$
\eta=H F(d \phi / d \theta)(d \theta / d H 1)
$$

Note that here no assumption is made about the respective orientations of $H F$ and of the electronic magnetisation ( $\phi$ and $\theta$ may be different).

The above enhancement factor is the one that would result when the RF field is directly applying a torque on the electronic magnetisation.

Ferromagnets are usually constituted by magnetic domains separated by domain walls (DW). If $d \theta$ is produced by a domain wall (DW) displacement, $d x$, under $H 1$ the enhancement factor can be written:

$$
\eta=H F(d \phi / d \theta)(d \theta / d x)(d x / d H 1)
$$


This enhancement is particularly strong $\left(10^{2}\right.$ to $\left.10^{5}\right)$ in DW centres of a ferromagnet and decreases from DW centres to domain centres.

The enhancement factor $\eta$ is much lower (10-100) in case of domain rotation for which

$$
d \theta / d H_{1}=1 / H a
$$

and

$$
\eta=(d \phi / d \theta)(H F / H a)
$$

where

$H a$ is the anisotropy field.

It is still lower in antiferromagnets (1-to 10) whete the only possibility for the electronic moments to rotate arises from the transverse suscepibility that is inversely proportional to the antiferromagnetic exchange stiffness.

From this description the enhancemat factor can be decomposed in macroscopic $\eta_{M}$ enhancement factor and in microscopic enhancement factor $\eta_{m}$.

For domain walls :

$$
\begin{aligned}
& \eta_{M}=\varepsilon_{d w}\left(d x / d H 1_{\text {max }}\right) \\
& \eta_{m}=H_{h f}(d \phi / d \theta)(d \theta / d x)
\end{aligned}
$$

And for domains:

$$
\begin{aligned}
& \eta_{M}=\varepsilon_{d}\left(d \theta / d H 1_{\text {max }}\right) \\
& \eta_{m}=H_{h f}(d \phi / d \theta)
\end{aligned}
$$


Where

$\varepsilon_{d}$ and $\varepsilon_{d w}$ represent the reduction of the electronic moment motion in the sample with respect to their maximum possible value.

$H 1_{\max }$ is the maximum RF field experienced in the sample.

Actually there is a spread of enhancement factor values within a sample, which results from the distribution of magnetisation orientation, of demagnetising field, etc. The $\varepsilon$ coefficients are distributed between 0 and 1 in the samples resulting in a distribution of the RF field power inside the sample.

The macroscopic enhancement factor can be written:

$$
\eta_{M}=\varepsilon \eta_{0}
$$

where

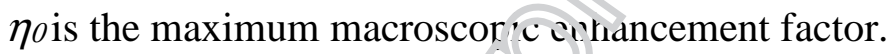

Since the factor $\mathcal{E}$ has various origins it can be decomposed in several contributions $\mathcal{\varepsilon}_{i}$ :

$$
\varepsilon=\varepsilon_{c} \varepsilon_{o} \varepsilon_{s d} \varepsilon_{d m} \varepsilon_{d}
$$

Where :

$\mathcal{E}_{c}$ : results from the field distribution inside the sample coil

$\varepsilon_{o}$ : results from the magnetic domain and domain walls orientation distribution with respect to the RF field direction 
$\varepsilon_{s d}$ : exists in the case of metallic samples. The RF field experienced by the nuclei decreases from the surface of the samples towards their centre.

The skin depth can be written:

$$
\delta=(2 \rho /(2 \pi \mu v))^{1 / 2}
$$

Where

$\rho$ is the samples resistivity

$\mu$ the sample permeability

$v$ the frequency

Considering typical values for me:ailic samples and a frequency of $200 \mathrm{MHz}$ (typical resonance frequency of Co na lei), $\delta$ is of the order of few microns.

$\mathcal{E}_{d m}$ : results from the reduction of the field experienced by the nuclei because of the demagnetisation field inside the sample. it therefore depends on the shape of the sample.

$\mathcal{E}_{d}$ : results from the effect of the domain walls motions. The magnitude of the domain wall motion will be small close to the defects pinning the domain walls while the motion of the domain walls will be large far from such defects. Therefore, $d x / d H 1$ will depend on the position of the probed nucleus inside the domain wall. [68]

All these coefficients are distributed between 0 and 1 . The shapes of these distributions are difficult to compute and in addition they will differ from one sample to another. However, some typical shapes are represented in figure 2.

As a consequence, the RF field experienced by a nucleus in a sample can be written: 


$$
\begin{aligned}
& H 1^{*}=\eta_{M} \eta_{m} H 1 \\
& H 1^{*}=\varepsilon_{c} \varepsilon_{o} \varepsilon_{s d} \varepsilon_{d m} \varepsilon_{d} \eta_{o} \eta_{m} H 1
\end{aligned}
$$

Since the $\varepsilon_{i}$ coefficients are distributed, $H 1 *$ is distributed also.

This decomposition of $\varepsilon$ is not limited to the above reported factors, additional sources of field reduction may exist depending on the nature of studied samples. Therefore, generalizing the above description, $\varepsilon$ is the product of a large number of independent positive variables:

$$
\varepsilon=\Pi_{i} \varepsilon_{i}
$$

Since the logarithm of a product is simply the sum of the logarithms of the factors. Thanks to the central limit theorem, the logarithm of a product of andom variables that take only positive values approaches a normal distribution. And as a censequence the product of these random variables approaches a log-normal distribution.

\subsection{FNR signal versus external RF field amplitude}

In the previous paragraph we have described the enhancement of the applied $H 1$ RF field resulting in the RF field $H 1$ * experienced by the nuclei. As already mentioned when the nuclei signal is measured it is also enhanced by the same enhancement factor. Taking both enhancement effects into account the measured signal of a nucleus for a given $H I$ and frequency $\omega$ can be written as (for simplicity the coefficients in equation 8 are omitted):

$$
s(H 1, \omega)=\eta(\omega) R\left(H 1^{*}\right)
$$

Where 
$R(H 1 *)$ is the nuclear spin response to $H 1^{*}$

Considering macroscopic and microscopic enhancement factor:

$$
s(H 1, \omega)=\eta_{M} \eta_{m} R\left(\eta_{M} \eta_{m} H 1\right)
$$

Now to compute the total FNR signal for a given $H 1$ and $\omega$ the nucleus signal has to be integrated over the whole range of $H I^{*}$

$$
S(H 1, \omega)=\int_{0}^{\infty} d\left(\eta_{m} \eta_{M H 1}\right) D\left(\eta_{m} \eta_{M} H 1\right) \eta_{m} \eta_{M} R\left(\eta_{m} \eta_{M} H 1\right)
$$

Where $D\left(\eta_{M} \eta_{m} H 1\right)$ is the distribution of $H 1^{*}$

Considering that $\eta_{0}$ is maximum macroscopic enhancerient factor possible in the sample we introduce the new variables $y$ and $y_{0}$ as:

$$
y=H 1 \eta_{M} \eta_{m}
$$

and

$$
y_{0}=H 1 \eta_{0} \eta_{m}
$$

With these new variable the distribution $D\left(\eta_{M} \eta_{m} H 1\right)$ can be expressed as:

$$
D\left(\eta_{m} \eta_{M} H 1\right)=\frac{d N}{d\left(\eta_{m} \eta_{M} H 1\right)}=\frac{d N}{\frac{d\left(\eta_{m} \eta_{M} H 1\right)}{\left(\eta_{0} \eta_{m} H 1\right)}\left(\eta_{0} \eta_{m} H 1\right)}=\frac{D\left(\frac{y}{y_{0}}\right)}{y_{0}}
$$

And the FNR signal equation 25 becomes:

$$
S(H 1, \omega)=\eta_{0} \int_{0}^{\infty} \frac{d y}{y}\left(\frac{y}{y_{0}}\right)^{2} D\left(\frac{y}{y_{0}}\right) R(y)
$$


If $y$ and $y_{0}$ are rewritten as:

$$
\begin{aligned}
& \ln (y)=x \\
& \ln \left(y_{0}\right)=x 0
\end{aligned}
$$

Equation (29) becomes:

$$
\begin{aligned}
& S(H 1, \omega)=\eta_{0} \int_{-\infty}^{\infty} \frac{\exp (2 x)}{\exp \left(2 x_{0}\right)} D\left(\frac{\exp (x)}{\exp \left(x_{0}\right)}\right) R(\exp (x)) d x \\
& S(H 1, \omega)=\eta_{0} \int_{-\infty}^{\infty} \exp \left(2\left(x-x_{0}\right)\right) D\left(\exp \left(x-x_{0}\right)\right) R(\exp (x)) d x
\end{aligned}
$$

Before continuing the development, a comment is requested about the use of distributions in the logarithmic space.

Let's consider the distribution $D(\varepsilon)=d N / d \varepsilon$ and the variable $x=\ln (\mathcal{E})$. The distribution of $x, \delta(x)$ can be written:

$$
d(x)=d N / d x=(d N / d \varepsilon)(d / d x)
$$

since $d \mathcal{E} / d x=\mathcal{E}$, it becomes

$$
\delta(x)=\varepsilon D(\varepsilon)=\exp (x) D^{\prime}(x)
$$

Where $D^{\prime}(x)$ is the distribution of $\mathrm{D}(\varepsilon)$ in logarithmic scale.

Using this formula in equation (33) it becomes:

$$
S(\log (H 1), \omega)=\int_{-\infty}^{\infty} \delta\left(x-x_{0}\right) \rho(x) d x
$$

where $\rho$ and $\delta$ are respectively the spin response and $H 1 *$ distribution in log scale. 
Actually the spin response $R$ is not a distribution but the formula is nevertheless formally applied to $R$.

As a result, equation 36 shows that, in a logarithmic space, the total nuclear spin response for a given $H 1$ and $\omega$ is actually the convolution product (assuming the distribution is symmetric with respect to $x_{0}$ ) of the $H 1^{*}$ distribution by the spin response, both taken in a logarithmic scale. For simplicity in the rest of the paper $S(\log (H 1), \omega)$ will be written as $S(H 1, \omega)$ but it will implicitly always be considered in a logarithmic space.

Before studying the shape resulting from this convolution product we have to consider the single spin response.

In our experiments we most often use spin echo sequences where the second RF pulse is applied twice longer than the first pulse $(\pi / 2-\pi$ sequence). At the resonance, using the matrix formalism developed by E.T. Jaynes [69] the spin respcise tor such pulse sequence can easily be written as:

$$
R(H 1, \omega)=\sin ^{3}(\gamma H 1 \tau)
$$

The representation of the spin response in log scale $\rho(x)$ is presented in figure $3 \mathrm{a}$. It is dominated by the first rotation towards $\pi / 2$. Increasing the field, the oscillations become faster and faster and will average out to zero in the logarithmic space. Narrow and wide distributions of $H 1^{*}$ are presented in figure $3 \mathrm{~b}$ and the convolution product of the spin response with the $H 1^{*}$ distribution is shown in figure $3 \mathrm{c}$. As can been seen, the resulting spin response is close to a Gaussian shape in a log scale (or a lognormal shape in a linear space). Similar field dependence of the FNR signal (although not represented in a log scale) has been reported in the work of M.B. Stearn [68]. This author focused on describing the response of domain walls while here a more generalized approach is proposed. 
As an example, in figure $3 \mathrm{c}$ the experimental data points obtained for a Co thin film $(6 \mathrm{~nm}$ thick) are represented on the same scale: the open circles correspond to the main line; the closed circles correspond to data recorded at lower frequency (interface range). In thin films the distributions of $\varepsilon_{i}$ are limited since the samples are thin enough to make the skin depth coefficient effect negligible and the films do not present domain walls. Because of these limited sources of RF field reduction, thin films are therefore the samples in which this generalized description of the FNR response might depart more significantly, the central limit theorem being more difficult to apply. Nevertheless, the data points follow nicely the spin response computed for the narrower of the $H 1 *$ distributions.

Finally, it can also be noticed that the width of the FNR signal follows the increase in width of the $H 1^{*}$ distribution. This will be discussed when computing the nuclear spins frequency density.

\subsection{Restoring field}

The above description focused on $\eta$ how ver, $\eta$ is a dimensionless coefficient that is difficult to compare to other magnetic entities like magnetic anisotropy or exchange energy etc. That is why we prefer to describe the enhancement of the RF field by a restoring field $H r$. This field characterises the torque experienced by the electronic magnetisation when it is slightly tilted from its position at rest by the RF field $H 1$. This restoring field can have any physical origin depending on the nature of the studied samples. For example, it takes the value of the anisotropy field for a uniaxial, single domain particle.

As already described the small oscillation amplitude of $H F$ results from the motion of the electronic magnetisation under the effect of the RF field $H 1$. We consider that when the 
electronic magnetisation is slightly tilted from its position at rest it experiences a restoring torque characterized by the restoring field $\mathrm{Hr}$ (figure 4). The tilt angle can be written as:

$\Delta \theta=H 1 / H r$

where

$\mathrm{Hr}$ is the restoring field traducing the restoring torque exerted on the electronic magnetic moments upon small orientation changes $\Delta \theta$.

The restoring torque exerted on the electronic magnetic moments is the same torque that is probed in ferromagnetic resonance - FMR - but here it is assumed that the electronic moments follow adiabatically the RF field, i.e. one is far from any electronic resonance. In other words, $1 / \mathrm{Hr}$ is proportional to the local transverse lecti nic susceptibility and will obviously be different in different materials. The RF oscilation of the electronic moments is in turn transferred to the hyperfine field $H F$, inducins its oscillating transverse component $H l^{*}$ that is directly responsible for the nuclear transitions. Hence, the value of $H 1 *$ becomes:

$$
H 1^{*}=H F . \Delta \theta=H 1(H F / H r)
$$

The ratio $H 1 * / H 1$ (equal to $H F / H r$ ) is the FNR enhancement factor $\eta$ as discussed in more detail above.

Using the restoring field description of the enhancement factor, equation 8 can be written:

$$
\begin{aligned}
& S\left(\omega, H_{1}\right)=K H F / H r R[\theta(H F / H r H 1)] \omega^{2} N(\omega) \gamma I(I+1) / T \\
& S\left(\omega, H_{1}\right)=K \omega^{3} / H r R[\theta(\omega /(\gamma H r) H 1)] N(\omega) I(I+1) / T
\end{aligned}
$$




\section{Computation of the nuclei frequency density}

\subsection{Log Normal model}

From equations 36 and 37 it follows that, in a single phase material, the signal intensity has a Gaussian dependence upon $\log (H 1 *)$. Since $H l^{*}$ is directly proportional to $H 1$ the signal intensity will also have a Gaussian dependence upon $\log (H 1)$.

$$
S(H 1, \omega)=K<\eta>\exp \left[-\log ^{2}\left(H 1 / H 1_{\text {opt }}\right) / 2 \sigma^{2}\right] \omega^{2} N(\omega) \gamma(I+1) / T
$$

Or if the restoring field description is used:

$$
S(H 1, \omega)=K \omega^{3} / H r \exp \left[-\log ^{2}\left(H 1 / H 1_{o p t}\right) / 2 \sigma^{2}\right] N(\omega) I(I+1) / T
$$

Where

$H 1_{\text {opt }}$ is the RF field value for which tie s1g nal is maximum,

$\sigma$ is the width of the Gaussian respense in $\log (H 1)$.

Experimentally $\sigma$ is found in the range 0.7 to 1.2 for single-phase samples, depending on the type of samples (thin films, particles, bulk samples etc.).

\subsection{Determination of the restoring field}

The maximum FNR signal is reached when the nuclear spins experience an effective RF field strength such that, after the pulse sequence, the nuclear magnetisation is perpendicular to $H F$. This happens when the average $H I^{*}$ reaches the value $H 1_{\text {opt }}{ }^{*}$ such that, for a single pulse of duration $\tau$ :

$$
H 1_{\text {opt }}{ }^{*}=\pi /(2 \gamma \tau) .
$$


Therefore, the value of the restoring field $\mathrm{Hr}$ can be written as:

$$
\begin{aligned}
& H r=(2 \tau / \pi) \gamma H 1_{o p t} H F \\
& H r=(2 \tau / \pi) \omega H 1_{o p t}
\end{aligned}
$$

Equation 46 shows that the restoring field is directly proportional to the optimum value of RF field $H 1$ for which the FNR signal is maximum. Since it is at the base of our data treatment process a detailed explanation is requested. It is described in figure 4 . First of all, it has to be kept in mind that for a specific nucleus and for a given pulse duration, the $H l^{*}$ value required to obtain the maximum of the FNR signal is a pre-known value (equation 44). As a consequence, if a sample (or one specific site in a sample) is magnetically stiff, the local electronic magnetization will experience a strong rest...ing tield (figure 4 top panel). Therefore, in order to fulfil, on this nucleus, the condition of equation (44) a large amplitude of the $H 1 \mathrm{RF}$ field has to be applied. On the contrary, if he smple (or one specific site in a sample) is magnetically soft, the local electronic reagerization will experience a weak restoring field (figure 4 bottom panel). Therefore, in order to fulfil on the nucleus, the condition of equation (44) a small amplitude of the $H 1$ RF field has to be applied.

In summary, the value of $H I$ for which the FNR signal is maximum is directly proportional to the restoring field.

\subsection{Role of the high-pass filter probe design in the determination of the restoring}

field.

As mentioned earlier in the paper, we use a broadband probe based on a simple high-pass filter design (see technical description further in this article) instead of a low-pass design (delay line). 
Within their pass-band, delay lines ensure frequency independent current through the coil, i.e. constant $H 1$. This might seem a need (and it is in nonmagnetic materials) but it may not be so in magnetic materials. The question is which of the external field $H 1$ or the enhanced field $H 1^{*}=\eta H 1$ should be kept frequency independent. Since $H 1^{*}$ is the actual field acting on the nuclei, the answer is $H l^{*}$.

As described earlier:

$$
\begin{aligned}
& \eta=H F / H r \\
& \eta=\omega /(\gamma H r)
\end{aligned}
$$

where $\mathrm{Hr}$ is the restoring field that defines the equil bium orientation of the local electronic moment. One sees that $\eta$ varies as $H F=\omega / \gamma$, is. incieases linearly with frequency. In the high-pass design of our probe, the voltage dron acress the coil is constant within the pass-band of the filter which means that the current a therefore $H 1$ decreases as $1 / \omega$ so that $H 1^{*}$ is frequency independent (if the magnetic nemeability, i.e. $\mathrm{Hr}$, is frequency independent). This discussion is rather academic because $h^{\prime}$ may be itself frequency dependent. For example, in metallic systems at low temperature, it is not uncommon that the magnetic permeability falls off as $1 / \omega$ in the FNR frequency range because of a large damping of the magnetic domain wall motion by eddy currents. However, the advantage of the high-pass probe scheme is that the frequency dependence of $\eta$ due to $H F$ is directly compensated and one is left only with the frequency dependence of $\mathrm{Hr}$, i.e. the information on the magnetic properties of the sample. In any case, this discussion shows the necessity to observe spectra for different $H 1$ strengths in order to measure $\operatorname{Hr}(\omega)$.

In the case of the high pass filter probe equation 46 can be written: 


$$
H r=\beta H 1_{o p t H P}
$$

Where

$H_{1 \text { opt } H P}$ is the optimum $H 1$ including the $1 / \omega$ frequency dependence of the $H 1$ in the high pass probe.

$\beta$ is a sample independent, constant, instrumental factor (calibrated against some sample where $H r$ is well defined) [1-3].

This simple relation allows to evaluate $H r$ and $\langle\eta\rangle$ in any sample, which is necessary in order to obtain the true nuclei density $N(\omega)$ out of the raw spectra. Moreover, FNR results can now be discussed in terms of restoring field, a material pros ty that is easily understood, rather than in terms of - instrument dependent - optimum excitaten field, or than in terms of enhancement factor - a notion for the specialists. Several evpriments have shown that the measured $\mathrm{Hr}$ is

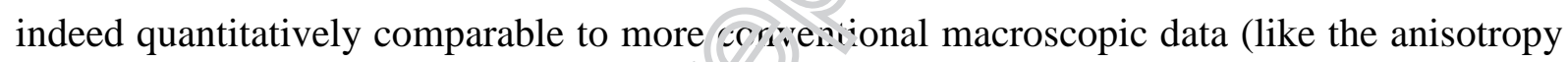
field, the coercive field or the exchange vias field) [1-3]. Therefore, we shall always express $H 1$ in units of $H r$ (i.e. we shall use $\beta H i$ instead of $H 1$ ).

Along the same line, and concerning the measured signal it may be argued that, while the sensitivity of the delay line to the nuclear induction is constant, that of the high-pass filter varies as $1 / \omega$. However, since the induced voltage in the coil is enhanced by $\eta$, the $1 / \omega$ sensitivity compensates for the $H F=\gamma \omega$ term in $\eta$. Then one is left only with the regular $\omega^{2}$ increase of sensitivity as in conventional NMR.

As a consequence, from equation 43 the FNR intensity as measured by the high pass probe, $S_{H P}(H 1, \omega)$, can be written:

$$
S_{H P}(H 1, \omega)=K \omega^{2} / H r \exp \left[-\log ^{2}\left(H_{1} / H_{1 o p t H P}\right) / 2 \sigma^{2}\right] N(\omega) I(I+1) / T
$$


It can be visualised by a set of data where the FNR signal intensity $S_{H P}$ is plotted as a function of the $H 1$ RF field frequency and amplitude. It can be plotted as a 3D picture or as contour curves like the ones shown in Figure 5 and figure 6, respectively. For such H1 2D mapping, the H1 amplitude is incremented linearly in log scale (see also data points in figure 3). Since the RF field strength is scaled as $\beta H 1$, then $H r$ is directly visualized and read as the position of the maximum signal intensity. The frequency variation of $H r$ versus the frequency is given by the locus of this maximum. For example, in figure 5 it can clearly be seen that while at high frequency the maximum of the FNR signal is obtained for a $\log (\beta H 1)$ value of the order of 1.8 , at lower frequency the maximum of the FNR signal is obtained for a $\log (\beta H 1)$ of the order of 1.4. Therefore, considering that each frequency range can be associated with a specific region of a composite sample, the $S_{H P}(\beta H 1, \omega)$ curves shed some light on the structural parameters governing, at atomic scale, the magnetic stiffness of the ferromagnet and the coupling strength between its components. Or simply speaking, (in $3 \mathrm{D}$ curves image directly the structural inhomogeneities, along the frequency axi (standard NMR structural information), and the magnetic in-homogeneities, along the $\mathrm{it}$ ticld axis (FMR like).

\subsection{Establishing the true Nuclei frequency density $N(\omega)$}

While the 3D $S_{H P}(H 1, \omega)$ surface allows to visualize simultaneously the structural and magnetic in-homogeneities, for quantitative analysis we prefer to split the FNR information in two 2D representations. The first $2 \mathrm{D}$ representation consists in building the true nuclear spins' frequency distribution. Since the response of the nuclear spins to the applied RF field has been mapped with a set of $S_{H P}\left(H 1_{\text {opt }}, \omega\right)$ curves, it is now straight forward to compute the density of nuclei versus the resonance frequency. From equation 50: 


$$
N(\omega)=\operatorname{THr} S_{H P}\left(H 1_{o p t}, \omega\right) / K / \omega^{2} /(I(I+1))
$$

Where

$S_{H P}\left(H 1_{\text {opt }}, \omega\right)$ is the maximum of the recorded FNR signal at a given frequency $\omega$.

In equation 51 the only parameter that has not been taken into consideration is the spread of enhancement (or of $\mathrm{Hr}$ ) at each frequency. In other words, the width of the $H 1 *$ distribution is not taken into account. It is clear that if this width is not taken into account $N(\omega)$ computed from equation 51 will have, for a given number of nuclei, a smaller value if the width of $H l^{*}$ is large than if it is narrow. Unluckily having a direct insight into the width of $H l^{*}$ is not possible. However, as shown in figure 3 even though $S_{H P}(H 1, \omega)$ is the convolution of the nuclear spin response by the $H 1 *$ distribution, the wirith of $S_{H P}(H 1, \omega)$ is dominated by the width of the $H l^{*}$ distribution. Therefore we consider the the width of $S_{H P}(H 1, \omega)$ provides an estimate of the $H 1 *$ distribution width. Therefore the + in $_{1}$ form for $N(\omega)$ becomes:

$$
N(\omega)=\operatorname{TH} \sigma S_{H P}\left(H 1_{o p t}, \omega\right) / \kappa_{1} /(I(I+1))
$$

Where

$\sigma$ is the spin response width in log scale.

From a practical point of view, a data treatment program has been built. From the $H 12 \mathrm{D}$ mapping $S_{H P}(\beta H 1, \omega)$ as shown in figure 5, Gaussian fits (in log scale) are performed at each frequency that allow computing $S_{H P}\left(\beta H 1_{o p t}, \omega\right), \operatorname{Hr}(\omega)$ and $\sigma(\omega) . N(\omega)$ is then computed following equation (52). An example of $2 \mathrm{D} N(\omega)$ spectrum is shown in figure 7 . This process allows accurate quantitative analyses of the different environment in the spectra (see examples part in this paper). In addition, while in bulk metallic alloys the absolute FNR signal depends on the samples' grain size, in the case of thin films and multilayers their thickness is thin 
compared to the RF field skin depth and absolute quantitative measurements can even be performed.

The second 2D output is the locus of the maximum of the FNR signal versus frequency, $H 1_{o p t}(\omega)$, or in other words since $H r=\beta H 1_{o p t}, H r(\omega)$. The frequency dependent restoring field $\operatorname{Hr}(\omega)$ recorded for the sample shown in figure 7 is shown in figure 8 . It can be seen that the sample has clearly 2 different frequency ranges with 2 different restoring fields. There is more than a factor of two between the smallest and the largest restoring field (and consequently more than a factor of 2 in term of enhancement factor).

Finally the last output, is the frequency dependent width of $S_{H P}\left(H 1_{o p t}, \omega\right), \sigma(\omega)$ (figure 9, for the same sample used for figure 8 and 9). This information is seldom exploited in itself but is required in order to compute $N(\omega)$.

The computation of $N(\omega)$ resulting from the FNR signal $H 12 \mathrm{D}$ mapping has become our standard data treatment procedure.

\section{Technical aspects}

\subsection{Double heterodyne spectrometer design}

The block diagram of the spectrometer that is currently in use is shown in figure 10. It is based on a double heterodyne design. The RF signal at $30 \mathrm{MHz}$ from the frequency generator is mixed up a first time with a $820 \mathrm{MHz}$ local oscillator. The resulting $850 \mathrm{MHz}$ wave is then down mixed with a $870 \mathrm{MHz}$ to $1600 \mathrm{MHz}$ signal provided by a frequency synthesizer. The resulting 20 to $750 \mathrm{MHz}$ signal is then amplified by a $10 \mathrm{~W}$ broadband power amplifier. The signal is sent via a fast pin diode SPDT switch to the broadband probe. After switching to the receiving 
measurement chain, the signal of the coil is amplified by a broadband low noise preamplifier and down mixed to $30 \mathrm{MHz}$ where the spin echo signal is integrated.

The role of the 2 frequency mixing is to reject outside of the spectrometer frequency range the spurious signals that might particularly result from the harmonics of the $30 \mathrm{Mhz}$ base frequency. The de-multiplexers allow measuring the echo along four different phases. This allows to perform the echo phase combinations explained further.

\subsection{FRN broadband high pass filter design probe head}

As mentioned earlier in the paper and shown in the spectrometer drawing in figure 10, we use a broadband probe based on a simple high-pass filtr acsign (figure 11) instead of a low-pass design (delay line). Beside the already mentioned aovantages and drawbacks, the high pass design has also practical advantages. First of ali, i: has the non-negligible advantage to be much more easily realized, using discrete elements, than a delay line. Furthermore, when dealing with magnetic materials, the modifications co fie inductance and quality factor of the coil due to the sample cannot be neglected. These modifications have a significant impact on the response of the probe close to its cut off frequency, be it high-pass or low-pass. For instance, if the inductance is multiplied by, say, a factor of 2 upon introduction of a high permeability sample, it means that, for a delay line with a high cut off frequency of $500 \mathrm{MHz}$, the frequency response will be significantly perturbed from $350 \mathrm{MHz}$ up, whereas for a high pass filter with a low cut off frequency of $20 \mathrm{MHz}$, the response will be affected only in the lower 14 - $20 \mathrm{MHz}$ range.

In the theoretical schematic diagram, if $\omega_{c}$ (in radians/s) is chosen as the low frequency cut off, $C, L$ and $R$ should take the following values:

$\mathrm{C}=1 / \mathrm{Z}_{0} \omega_{c}$ 
where

$\mathrm{Z}_{0}$ is the matching impedance (usually $50 \Omega$ ).

Actually, in our spectrometer, the coupling capacitors in the SPDT switch cannot be neglected and it has been taken into account together with the feeding line between the switch and the probe. Such slight modifications to the theoretical design must be considered on a case by case basis but they only affect a narrow frequency range in the lowest part of the pass-band which, anyhow, is also dependent on the sample and the fillin sactor. Practically, above $2 \omega_{c}$, the probe can be considered as a pure matching resistance $P$. Whith constant voltage across it and across the coil in parallel with it.

For our most standard probe, $\omega_{c}$ has been ixf at $20 \mathrm{MHz}$. The corresponding values of the components are:

$$
L=200 \mathrm{nH}, \quad \mathrm{C}=150 \mathrm{pF}, \quad R=50 \Omega
$$

We routinely use a coil that is 7 turns of silver ribbon $0.7 \mathrm{~mm}$ wide, wounded on a $10 \mathrm{~mm}$ long, $6 \mathrm{~mm}$ diameter frame (for multilayers and thin films a coil with a rectangular section of similar area is better suited). The use of ribbon wounded with a significant gap between turns helps to avoid, as much as possible, stray capacitances between turns which inevitably introduce a high frequency cut off.

Of course higher values of the cut off frequency can be chosen if the low frequency range is not interesting. As a rule of thumb, raising the cut off frequency by a factor $\alpha$ will increase the 
sensitivity by a factor $\sqrt{ } \alpha$ (for the same coil length and diameter) and $H 1$ by the same factor (for the same RF power) which may be quite useful.

Since we are usually working at liquid helium temperature, metal film resistors have been selected for $R$, which do not vary with temperature (less than $1 \%$ change between $4 \mathrm{~K}$ and 300 K). They must be chosen not to be inductive. Some metal film resistors are etched in such a way that the film forms a 1 to 3 turns coil. Then it is better to raise a low valued resistor (10 or $20 \Omega$ ) to the desired $50 \Omega$ value by removing part of the metal film. Alternatively, higher valued

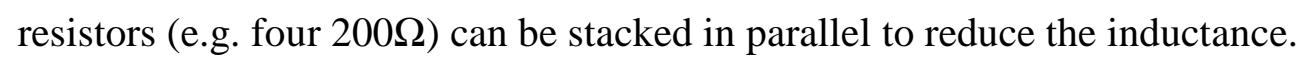

\subsection{Operating mode}

\subsubsection{Pulse sequence}

All our experiments are performed with the echo technique and most of the time we use the very simple $\pi / 2-\pi$ sequence where the second pulse is twice longer than the first one. We usually use pulse length of $3 \mu$ s and $6 \mu$ s separated by a delay time that can be as short as $2 \mu s$. The use of very short delay times minimises the effect that could result from a frequency dependent variation of the transverse spin-spin relaxation time $\left(T_{2}\right)$ on the shape of the FNR signal. It is because of the use of short delay times that a fast SPDT switch is required in the set up. This is one of the limiting feature in our set up design since fast SPDT switches able to handle RF powers of the order of $10 \mathrm{~W}$ are not commonly found. However, in ferromagnets usually $10 \mathrm{~W}$ is large enough for most of the samples, and if the RF power is not strong enough to reach a $90^{\circ}$ rotation of the spins, the pulse length can be increased. In such case we simply double our standard pulse length what makes possible to explore samples with restoring fields twice stronger compared to our standard experimental conditions. 
One of the main feature in the experimental setup is the possibility to apply $H I$ and to measure the signal along any of the four directions $(\mathrm{X}, \mathrm{Y},-\mathrm{X},-\mathrm{Y})$ of the rotating frame. It permits the use of phase shifted pulse sequences to get rid of most spurious signals while accumulating a spin echo signal. The rather well known set of phase shifted pulse sequence $(+\mathrm{X},+\mathrm{X}),(+\mathrm{X},+\mathrm{Y})$, $(+X,-X),(+X,-Y)$, generates echoes successively in the $+Y,-Y,+Y,-Y$ directions. Once accumulated with the correct sign, the corresponding echo signal is free from the FID, the ringing and cross-talking signals due to and in phase with the second pulse and also from any DC offset. The reason for getting rid of the FID signal is that it is opposite to the echo signal; experimentally we have observed increases of up to $30 \%$ of the output signal by using such set of sequences for the short delays ( 2 to $5 \mu \mathrm{s}$ ) we use between the two pulses. Actually, in our standard spin-echo measurement procedure, we like to 're the set of two pulse sequences shown in table I which combines phase rotation of the sec nd pulse with phase rotation of the first pulse. Such set allows a further reduction of the ring due to the first pulse. The order of the pulse sequences is such that it generates which not only cancels DC offset but ino reduce linear and parabolic drifts of this offset. This is important when accumulating for days. The phase coding of the echo provides also some additional rejection of broadcast signals (combined of course with a proper shielding of the sample probe). This is particularly necessary when scanning the frequency in the crowded 30$150 \mathrm{MHz}$ range.

Finally the phase information is also taken into account when establishing the $N(\omega)$ nuclei density so that there is no base line offset resulting from the computation of the signal modulus. Zero signal will therefore result in an average zero value of $N(\omega)$ (i.e. random phase).

\subsubsection{Fast scanning mode}


Prior to 2010 the frequency scanning of the spectrometer was performed by a PLL (Phaselocked loop) frequency synthesizer. With such synthesiser the frequency switching is slow (hundreds of ms), much larger than the longitudinal relaxation time $T_{l}$ (few ms) of most of the studied samples. It is therefore not interesting to switch frequency after each data acquisition sequence. In consequence, data acquisition was performed by averaging measurements at a given frequency during typically $500 \mathrm{~ms}$, performing an integer number of the 16 phase permutations shown in table 1 . Then the frequency was switch to the next one and the same averaging process started again. To further supress any long time scale drift, the spectra were scanned successively with increasing and decreasing frequency steps.

While this process produces reliable measurements, after each single frequency measurement sequence it is necessary to wait until the whole nuclearspin $y$ stem returns to equilibrium before performing a new measurement (several times $i$, Considering that a typical $\pi / 2-\pi$ pulse sequence and the following spin echo integraten $\mathrm{t}$ k kes about $20 \mu \mathrm{s}$, while the $T_{1}$ relaxation time in ferromagnetic samples is at least of th oric of $1 \mathrm{~ms}$, the experimental set up working cycle was very small (at most $1 / 50$ even for samples having a $T_{1}$ as short as $1 \mathrm{~ms}$ ). With the advent of fast switching frequency synthesizers (particularly thanks to DDS : Direct Digital Synthesis) it was possible to develop a faster scanning mode. Indeed, with frequency synthesizers able to switch frequency in less than $1 \mu$ s their switching time becomes smaller than the measurement sequence time.

Since 2010 the setup is built with such fast switching frequency synthesizer allowing frequency switching in less than $1 \mu$ s. It allowed to develop a new scanning mode. Rather than averaging FNR measurements at each individual frequency and therefore having to wait at least $T_{1}$ between each measurement, once a measurement is performed at a frequency F1 it is switch to the next frequency F2 without waiting until the nuclear spin system returns to equilibrium. 
Actually the whole spectrum frequency range can be scanned with fast frequency scanning without waiting until the nuclear spins return to equilibrium at each frequency. It is the spectral hole burning concept. It is only after the scanning of the whole frequency range (typically 200 to 300 data points) that the recording is stopped and the whole nuclear spin system is allowed to return to equilibrium. For a sample with a $1 \mathrm{~ms} T_{1}$ this fast accumulation mode allows 50 times more data measurements than the classical (slow) data acquisition mode. Actually the longer is the sample's $T_{1}$ the more efficient is the method. As for the previous "slow mode", permutations of phases and up and down frequency scans are always performed.

The only problem that can be encountered with the fast scanning mode originates from the spin diffusion effect. Indeed, the frequencies have to be scanned at a speed faster than the spin diffusion. However, if spin diffusion is an issue, it sal be solved by simply increasing the frequency distance between the measurement ste $s$. This is exemplified in figure 12 where the rather narrow spectrum of bulk Co has bef. scanned with different frequency steps. The reference spectrum has been scanned usir the egular slow mode with $40 \mathrm{kHz}$ steps. The fastest frequency scanning has been performed in 2 fast scans (shots) using the $80 \mathrm{kHz}$ steps (the second being shifted by $40 \mathrm{kHz}$ with respect to the first one in order to record the same number of data points as in the slow mode. If more shots are required they are successively shifted by $40 \mathrm{kHz}$ ). It can be seen that, because of the spin diffusion, the FNR signal amplitude of the fastest scan is significantly reduced compared to the spectrum obtained with the slow acquisition mode. However, since phase permutations and up and down frequency scans are always performed, the shape of the NMR spectrum is not altered by the spin diffusion effect. For frequency steps of $400 \mathrm{kHz}$ and above the decrease in the FNR signal intensity becomes negligible. Actually in most cases the spectra are much more widely spread in frequency, requesting the use of rather wide frequency steps, therefore the effect of spin diffusion has seldom been detected. 
From the data in figure 12, the gain in time obtained with the fast accumulation mode has been estimated by considering the same resulting signal/noise ratio. The largest gain in time is obtained for fast scanning with 200 and $400 \mathrm{kHz}$ steps (or 5 and 10 shots respectively) for which the fast scanning mode is 24 times faster than the slow scanning mode. Since the fcc Co sample used in these tests has a rather narrow FNR spectrum this can be considered as a worst case. In practice, using similar total accumulation times as for the slow acquisition mode, the fast acquisition mode allowed to perform typically 100 times more frequency scans resulting in an increase in sensitivity of the order of a factor of 10 compared to the slow scanning mode.

It is this fast scanning mode that has been used for our most recent studies.

\subsubsection{Performances in the fast sc.mning mode}

With the architecture described in this paper ti- requency response of the FNR spectrometer has been adjusted to be flat within $0.5 \mathrm{eB}$ in the frequency range from 20 to $750 \mathrm{MHz}$. Since Co thin films are the most challeng1t: : pe of samples that are studied they can be used to estimate the sensitivity of the spectrometer. Co films are measured at $1.8 \mathrm{~K}$, typically during 12 hours' accumulation times resulting in the averaging of about $10^{6}$ spectral measurements. The films surface area is usually of the order of 1 to $5 \mathrm{~cm}^{2}$. With these experimental conditions and using the fast scanning mode, the set-up sensitivity is better than 0.1 atomic layer of Co $(0.02$ $\mathrm{nm}$ of Co or $5.10^{14}$ Co atoms) for samples having an enhancement factor of the order or 4000 $(H r=0.005 T)$. Of course the sensitivity becomes worse with the increase of the restoring field (i.e. decrease of the enhancement factor). For example, the sensitivity would be of $10^{17} \mathrm{Co}$ atoms for samples having a $1 \mathrm{~T}$ restoring field. 


\section{Experimental examples}

Combining the fast spectrometer scanning mode and the H1 2D mapping methodology allowing the determination of the true frequency density of the nuclei also made possible the development of new investigation strategies. The examples presented in this chapter will focus on these new approaches. More standard studies can be found in the other references at the end of the paper. In the first example we show that in the study of thin Co films absolute quantitative analyses can be performed by FNR. In addition, the improved sensitivity acquired by the fast scanning mode also made possible the investigation of single buried interfaces and allowed to obtain a very unique insight into the morphology and magnetic properties of interfaces. The second example focuses on assemblies of Co particles. For this study we introduced temperature

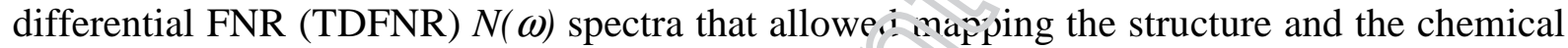
order within the size distribution of assemblies of paricles. Finally, we will close this part with preliminary results obtained on samples consi:uter of interacting particles. This is a work under progress and although the behaviour of the $5 \cdot \mathrm{R}$ spectra is not completely understood yet, it opens the path for a new investigatici fie 1.

\subsection{Co/organic thin films and investigations of single buried interfaces}

Motivated by the development of spintronic, magnetic thin films and multilayers have been extensively studied. Their interfaces' structure and morphology play a very important role in the magnetic and transport properties of such films. That is why they have been the subject of several FNR studies. While the role of interfaces is important when building inorganic systems, their influence becomes even stronger when one of the components is an organic material. In this framework we have recently studied Co/zinc tetra-phenyl porphyrin ( $\mathrm{Zn} / \mathrm{TPP}$ ) heterostructures $[49,50]$. When studying Co thin films, the basic investigation method consists 
in studying a series of samples as a function of the Co thickness. In the case of Co films the thickness of the samples is much thinner than the RF field $H 1$ skin depth. Therefore, the FNR spectra represent the contribution of all the Co atoms inside the sample and absolute FNR intensities can be obtained when using the described $H 1$ 2D mapping method to compute $N(\omega)$. The best way to show this is first to normalize the FNR spectra to the samples' surface area so that any discrepancy in their size and shape is removed. No other sort of normalization of the FNR spectra has been used. With such normalization the integral intensity over the full FNR spectral range should increase linearly with the increase of the thickness of the Co films. An example of such plot is represented in figure 13 for a series of ZnTPP/Co/ZnTPP sandwiches. As expected, the FNR $N(\omega)$ integral intensity increases linearly with the increase of the deposited Co thickness. The error bars that are shown wigure 13 are mostly due on one hand to the uncertainties in the deposited Co thickness (tink ness error bars) and on the other hand to the uncertainties in the measurement of the sampies' surface area $(N(\omega)$ error bars, few \%) and not from the FNR measurements. Sucl lin of plot is particularly interesting for revealing the presence of non-ferromagnetic $\mathrm{Co} a r$ ni $\mathrm{Indeed}$, since the measurements are performed in zero external static field the nonmagnetic Co atoms will vanish from the FNR intensity. This is why the linear fit of the FNR intensity shows that the $N(\omega)$ integral intensity does not extrapolate to zero for zero Co thickness. From the intercept of the linear fit, the amount of Co that is no longer ferromagnetic can be determined. About $1.4 \mathrm{~nm}$ of Co are not ferromagnetic at $1.8 \mathrm{~K}$ (temperature of measurements) and hence not detected in the FNR signal.

Because of the very different nature of the materials that are involved in such hybrid Co/organic films it is very likely that the interfaces on both sides of the Co films show very different morphologies and properties. One way to probe such interface asymmetry is to study in situ the morphology of thin films during their first stage of growth. However, such type of study does not allow to ascertain, once the samples are fully grown, that the buried interfaces present the 
same morphology as when exposed to the free surface. Very few experimental techniques make possible the study of the morphology of such buried interfaces. In addition, even if it is possible top and bottom interfaces can hardly be distinguished. All these limitations are particularly true when post processing of the samples has to be avoided as in the study of Co/organic heterostructures where the two materials have very different mechanical properties. To probe such buried interfaces, and making use of the increased sensitivity resulting from the fast scanning mode, we have developed an original approach in order to study individually the top and bottom interfaces of Co films in contact with another nonmagnetic element. For this purpose, heterostructures with specific architectures were prepared: Fe/Co/ZnTPP and ZnTPP/Co (6nm)/Fe. Such architectures allow to split the contributions of the two buried Co interfaces in two distinct frequency ranges allowing the ivestigation of single buried interfaces. Indeed, when $\mathrm{Co}$ atoms have $\mathrm{Fe}$ atoms amone their nuarest neighbours, the Co resonance frequency increases $[6,8]$ and thus the $\mathrm{Co} / \mathrm{Fo}$ interface contributions will appear at high frequency. In contrary the low frequency critishitions will originate from the single Co/ZnTPP or ZnTPP/Co interfaces only. The $N($ rol spectra of these two samples are shown in Figure 14. Clearly the low frequency intensities ot the spectra are not superimposed showing that the bottom and top interface morphologies are different. A much larger amount of Co atoms is involved when Co is deposited on top of ZnTPP than when ZnTPP is deposited on top of Co. As can be seen the shape of the low frequency spectra are structure less therefore it looks rather difficult to develop interface morphology models as it has been done for inorganic films. However, using the intensity study in figure 13 quantitative information can be gathered from the interface integral intensity. Indeed, the slope of the $N(\omega)$ integral intensities versus the deposited Co thickness, Figure 13, corresponds to the FNR experimental intensity per nanometre of Co. Using this information, the amount of Co atoms involved in the interfaces' frequency ranges of the $N(\omega)$ spectra can be easily computed. It can be estimated that when 
ZnTPP is deposited on top of Co, the thickness of Co involved in the interface is of the order of $0.4 \mathrm{~nm}$ of equivalent Co thickness while when Co is deposited on top of ZnTPP, it is of the order of $0.7 \mathrm{~nm}$ of equivalent Co thickness. It clearly shows that the two Co interfaces are asymmetrical. The interface resulting from the deposition of Co on top of ZnTPP being much thicker than the interface resulting from the deposition of ZnTPP on top of Co. This example also illustrates the sensitivity of the experimental set up. Indeed, $0.4 \mathrm{~nm}$ of Co represent 2 atomic layers of Co. As can be seen the signal to noise ratio is very satisfactory especially if considering that the contribution of these two atomic layers of Co is spread over a frequency range of about $150 \mathrm{MHz}$.

As explained in the first part of the paper the second input that can provide the $H 12 \mathrm{D}$ mapping method is the restoring field experienced by the probea atoms. In the case of thin films, the restoring field $(\mathrm{Hr})$ can be identified to the in plan magnetic anisotropy field of the samples. Figure 15 shows the frequency dependent rstoring field obtained on both single Co/ZnTPP and ZnTPP/Co interfaces. The behaviour (N) tho samples is similar as the largest restoring field is obtained at $224 \mathrm{MHz}$. It is cle e to the frequency of bluk hcp Co atoms and therefore is attributed to the Co atoms situated far from the interfaces. The low frequency $(<200 \mathrm{MHz})$ restoring fields are assigned to the Co atoms at the Co/ZnTPP and ZnTPP/ interfaces and are typically twice smaller than the restoring field of the bulk Co atoms. It shows that the restoring field of the interfaces is significantly smaller than the restoring field of the bulk of the layers and reflects the behaviour of the local magnetic anisotropy inside the sample. It can also be noticed that there is an overall shift of the samples' restoring field. The sample in which Co is deposited on top of ZnTPP is magnetically softer than the sample in which ZnTPP is deposited on top of Co. The increased interface mixing originating from the deposition of Co on top of ZnTPP is probably at the origin of an overall decrease of the average restoring field of the sample. Finally it can also be noticed that while the two samples show a difference in restoring 
field of the order of $30 \%$ (figure 15), the $N(\omega)$ spectra (figure 14) have the same total integral intensity since the Co films have the same thickness. This is obtained through the computation of $N(\omega)$ since as in figure 13 no additional normalisation is performed apart dividing the FNR signal by the surface area of the sample.

It is the first time that this concept of splitting interface contributions in separate frequency ranges was used. It can be applied to any type $\mathrm{Co} /$ nonmagnetic element interface.

\subsection{Temperature Differential Ferromagnetic Nuclear Resonance Spectra (TDFNR)}

Assemblies of nano-objects are investigated in many leearch fields from physics to medicine. However, since it is often difficult to produce ohjcts with mono-dispersed sizes, identifying the key parameters enhancing their efficiency is blurred because they show wide size distributions. The accuracy of the FNR presented in this paper made possible in introduce the concept of Temperature Differential Ferromagnetic Nuclear Resonance (TL: $\sqrt{ } R)$ Spectra that allow sampling the crystallographic structure, the chemical composition and the chemical order of non-interacting ferromagnetic nano-objects within specific size ranges of their size distribution [54]. This methodology consists in measuring the FNR $N(\omega)$ spectra of a given sample for different temperatures and establishing the true nuclei frequency density with equation 52. As already mentioned in the previous paragraph, when performing FNR in ferromagnets, an FNR signal can only be obtained if the magnetization direction of the probed atoms is fixed (blocked) during the FNR measurement time scale. In the case of nanoparticles, for a given volume, their magnetization is not blocked any-more above the so called blocking temperature. Above this temperature the particles become superparamagnetic and their contributions vanish from the FNR signal. In 
consequence FNR spectra only involve the particles with blocking temperatures larger than the FNR measurement temperature. Using this concept the temperature differential FNR (TDFNR) $N(\omega)$ spectra consist, after having measured FNR spectra for specific temperatures (T1, T2, T3, T4 etc. in increasing temperature order) in computing the difference of the spectra recorded for adjacent temperatures (FNR(@T1 minus FNR@T2, FNR(@T2 minus FNR@T3, FNR(@T3 minus FNR@T4...) The resulting TDFNR $N(\omega)$ spectra represent the spectra of the particles for specific size ranges only, within their size distribution. An example is given in figure 16. In figure 16a the FNR spectra recorded at 1.8, 4.2, 77K are shown. Since the measurements are performed on the same sample no normalization has been performed and figure 16a shows the FNR $N(\omega)$ as computed from equation 52. It can be seen that around $217 \mathrm{MHz}$ all the spectra are superimposed whatever the measurement temperaturc This is expected since this frequency range corresponds to the large multi-domain fcc co raticles that stay in a ferromagnetic state whatever the measurement temperature. The strati single domain particles will appear at higher frequencies because of the additional co trioutica of the demagnetizing field (equation 3). Indeed, the demagnetization field is ine opposite direction to the electronic magnetization direction and is therefore pointing in the same direction as the hyperfine field. Therefore, in single domain particles the field experienced by the nuclei is increased (and in consequence the resonance frequency also) compared to the field experienced by the same nuclei inside a multidomain sample. The TDFNR spectra resulting from the spectra differences are shown in figure 16b. The detailed analysis of the TDFNR spectra shows that while the smallest particles are constituted by a mixture of fcc and hcp Co particles, the largest one (>10 $\mathrm{nm})$ are dominated by fcc particles. In addition, the TDFNR line intensities allow estimating the size distribution of the nano-particles [54]. Therefore, the method provides a very complete and unique insight into the structure, chemical order and size distribution of assemblies of nano-objects. 


\subsection{Magnetic dipolar interactions in assemblies of nano-objects}

The previous work has been performed in samples with particles that are statistically far enough from each other so that they do not interact magnetically through dipolar interactions. This is requested for the validity of the investigation method. However, studying the magnetic dipolar interactions taking place when the particles become close enough is of high interest both from a fundamental point of view as for applications. Reducing the average distance between the particles has been obtained by increasing the Co content in samples similar to the previous ones. An example of FNR $N(\omega)$ spectra recorded at 1.8, 4.2, and $77 \mathrm{~K}$ is shown in figure 17a. As can be seen the results are unexpected. Indeed, as the temperature increases the FNR $N(\omega)$ spectra increase in intensity. Not only it seems that the paramagnetic particles do not vanish from the spectra when the temperature is increased, but in ariaition, the number of observed Co atoms increases with the measurement temperature. $\operatorname{Tr} 3 \exp ^{\prime}$ anation of this surprising behaviour can partially be found in the temperature dependences the restoring field of the samples shown in figure $17 \mathrm{~b}$. While in the case of the isolat $\mathrm{i}$ pat icles the restoring field did not vary significantly with the measurement temperatures, in the case of the interacting particles the restoring field decreases significantly when the measurement temperature is decreased and shows a liner behaviour in $1 / \mathrm{T}$ (inset figure $17 \mathrm{~b}$ ). The decrease of the magnetic anisotropy in interacting particles is a known effect [70]. It is explained the following way: when the temperature decreases, the interactions between the particles result in a collective behaviour of the nanoparticles. This is described by an effective magnetic volume involving a larger and larger number of particles when the temperature is decreased. The increase of the number of particles in the effective magnetic volume results in a decrease in the effective magnetic anisotropy. This effect explains the decrease of the restoring field when the measurement temperature is decreased. While the behaviour the restoring field is qualitatively understood, we still do not understand why the $H 12 \mathrm{D}$ mapping method used to establish the FNR $N(\omega)$ spectra fails to 
provide satisfactory intensities. It is probably due to some collective effect of the particles involved into the effective magnetic volume. It may also be due to a temperature dependent onset of an antiferromagnetic ordering of some of the particles. These particles would experience a significantly stronger restoring field which was not probed in the range of the $\mathrm{HI}$ 2D mapping used in this study. This work is still under progress.

\section{A broadband FNR spectrometer with automated frequency scanning built from on the shelf components}

During the last decade numeric electronics has seen a huge development and new kinds of electronic components became available. Therefore, we ecently started to develop a new spectrometer based on the AWG/UHFLI solutior of ? wich instruments. The block diagram of the very simple set up is shown in figure 18 The $4 W G$ allows producing the pulse shapes in frequency ranges from DC to $600 \mathrm{MHz}$. The iw, ses are amplified by a wide band $10 \mathrm{~W}$ amplifier and sent to the broadband probe via a $1 \mathrm{st}_{\mathrm{P}}$ in diode switch (with $50 \mathrm{~dB}$ isolation in the requested frequency range). The sample signal is then amplified by a low noise preamplifier and sent to the UHFLI input where a homodyne detection of the signal is performed. An example of FNR signal recorded with this set up is shown in figure 19. It represents the signal obtained with one single frequency scan recorded in slow mode. The sample and probe used for this measure is the same as in figure 12. The RF power used for figure 19 was smaller than the one used for figure 12, and explains why the high frequency line is larger than in figure 12. Considering that each frequency point of the spectra in figure 12 correspond to the averaging of 4000 measurements, a similar averaging time performed with the AWG/UHFLI set up would result in an improvement in the signal to noise ratio by a factor of 60 . The performances of the set up look promising. The fast scanning mode has not been implement yet, but the AWG/UHFLI 
performances should make it possible. We hope that the possibility to build automated broadband FNR spectrometer with such simple architecture will make FNR available to a much broader scientific community.

\section{Conclusion}

Because of its particularities, NMR in ferromagnets (ferromagnetic nuclear resonance, FNR) is not very widely used. This is mainly due to the two main particularities of NMR in ferromagnets. The first one is that even without applying any external magnetic field the nuclei already experience a magnetic field that has for origin the spontaneous magnetisation of the samples. Therefore, FNR spectra have to be recordea i,y frequency scanning. The second particularity of FNR is that the radiofrequency $R \Gamma, 1 \in$ la experienced by the nuclei is not the RF field applied to the sample but is a field enhar cec by the electronic magnetic susceptibility of the sample. The first consequence of this ent ancement is that the optimum FNR excitation power required to measure the spectr is not known and has to be determined for each sample. The second consequence results from the fact that since the magnetic susceptibility might differ from one nucleus site to another within the same sample, the recorded spectrum for a specific RF field power might not reflect the true nuclei frequency distribution, making difficult a quantitative analysis of the data. In this paper we have presented in detail the $H 12 \mathrm{D}$ mapping methodology that we have developed to overcome the difficulties resulting from the particularities of FNR. It is presented both from a conceptual point of view as from an experimental point of view. Particular emphasis has been put on the use of the high pass filter design for the broadband probe and on the fast scanning mode developed in our most recent spectrometer. The proposed approach allows to map the FNR signal for a wide range of $H 1$ power. From the 2D mapping of the FNR signal it is possible to determine the site dependent 
enhancement factor of the RF field and correct the FNR response in order to establish FNR spectra that reproduce the true nuclei frequency density. In addition, the determination of the site dependent RF field enhancement factor (rewritten in a form similar to an effective magnetic anisotropy field) gives a unique insight into the local magnetic properties inside the studied samples. Examples of recent experimental results where this approach has been particularly efficient has been given. It has been shown that absolute FNR intensity measurements can be performed on Co thin films and that the experimental set up sensitivity is high enough for investigating single buried interfaces. Finally, the paper is concluded with the proposition of a design for an FNR spectrometer based from on the shelf electronic components. It will allow to make FNR set ups available to a much broader scientific community.

\section{Acknowledgements}

Part of this work was funded by the Fran wational Research Agency (ANR) through the Programme d'Investissement d'Aver ir uder contract ANR-11-LABX-0058_NIE within the Investissement d'Avenir program ANR-10-IDEX-0002-02. 


\section{References}

1. C. MENY AND P. PANISSOD

Nuclear Magnetic Resonance in Ferromagnetic Multilayers and Nanocomposites: Structure Magnetic Properties and Magnetic Interactions;

Springer International Publishing AG (2017)

G.A. Webb (ed.),

Modern Magnetic Resonance, https://doi.org/10.1007/978-3-319-28275-6_139-1

2. P. PANISSOD, C. MENY

Nuclear Magnetic Resonance investigations of the structure and magnetic properties of metallic multilayers and nanocomposites

Appl. Magn. Reson. 19, 447-460 (2000)

3. P. PANISSOD, C. MENY, M. WOJCIK, E. JEDRYKA

Magnetic properties and structure of metallic multilayers investigated by NMR

Materials Research Society Spring Meeting, San Francisco, USA, 30 Mars - 4 Avril 1997

Mater. Res. Soc. Symp. Proc. 475, 157-168 (1997)

4. S. COLIS, D. STOEFFLER, C. MENY, T. FIX, C. LEUVREY, G. POURROY, A. DINIA, AND P. PANISSOD

Structural defects in $\mathrm{Sr}_{2} \mathrm{FeMoO}_{6}$ double perovskite: Experimental versus theoretical approach

J. Appl. Phys. 98, 033905 (2005)

5. S. COLIS, G. POURROY, P. PANISSON, C MENY, AND A. DINIA

Correlation between magnetic properties ana nuclear magnetic resonance observations in $\mathrm{Sr}_{2} \mathrm{FeMoO}_{6}$ double perovskite

J. Magn. Magn. Mater. 272, 2018-2020(2004)

6. M. MALINOWSKA, C. MENY, E, JCIR YKA, P. PANISSOD

The anisotropic first-neighbour con, il ution to the hyperfine field in hexagonal-closepacked Co: A nuclear magnetir resnance study of diluted alloys and multilayers

J. Phys.: Condens. Matter. 1C.49'9-4928 (1998)

7. J.P. JAY, M.WOJCIK, P. PANiS SOD

Hyperfine field and ordering in bcc CoFe bulk alloys studied by ${ }^{59} \mathrm{Co}$ NMR and MonteCarlo simulation

Z. Phys. B 101, 471-486 (1996)

8. C. MENY, E. JEDRYCKA, P. PANISSOD

Satellite structure of Co NMR spectra in some Co alloys

J. Phys. : Condens. Matter 5 1547-1556 (1993)

Hard Magnets

9. H. NAKAMURA, T. WAKI, Y. TABATA, C. MENY

Co site preference and site-selective substitution in $\mathrm{La}-\mathrm{Co}$ co-substituted magnetoplumbite-type strontium ferrites probed by ${ }^{59} \mathrm{Co}$ nuclear magnetic resonance J. Phys.: Mater. 2, 015007 ( 2019 )

10. H. NAKAMURA, A. SHIMODA, T. WAKI, Y. TABATA, C. MENY

Site-dependent cobalt electronic state in La-Co co-substituted magnetoplumbite-type ferrite: Co-59 nuclear magnetic resonance study

J. Phys.: Condens. Matter 28346002 (2016)

11. AZIZI, A. SAHARI, M.L. FELLOUSSIA, G. SCHMERBER, C. MENY, A. DINIA

Growth and properties of electrodeposited cobalt films on Pt/Si( 100$)$ surface

Appl. Surf. Sci. 228, 320-325 (2004) 
12. S. COLIS, A. DINIA, C. ULHAQ-BOUILlET, P. PANISSOD, C. MENY, G. SCHMERBER, AND J. ARABSKI,

Magnetic, transport and structural properties of Co/Ir multilayers grown by molecular beam epitaxy

Phys. Stat. Sol. A 199, 161 (2003)

13. M. DEMAND, M. HEHN, R.L. STAMPS, C. MENY, K. OUNADJELA

Structure and magnetic properties of epitaxial cobalt islands

Eur. Phys. J. B 25, 167-176 (2002)

14. F. BENSMINA, A. DINIA, C. MENY, P. PANISSOD, D. MULLER

New ternary ferromagnetic phase induced by annealing at the $\mathrm{FeMn} / \mathrm{Co} / \mathrm{FeMn}$ interfaces

J. Magn. Magn. Mater. 226-230, 473-475 (2001)

15. S. COLIS, A. DINIA, P. PANISSOD, G. SCHMERBER, C. MENY

Biquadratic coupling in Co/Ir multilayers and sandwiches deposited by ion beam sputtering (IBS)

J. Magn. Magn. Mater. 226-230, 1725-1727 (2001)

16. A. MICHEL, V. PIERRON-BOHNES, J.P. JAY, P. PANISSOD, S. LEFEBVRE, M. BESSIERE, H.E. FISCHER, G. VAN TENDELOO

Stabilisation of $\mathrm{fcc}_{\mathrm{C}}$ cobalt layers by $0.4 \mathrm{~nm}$ thick manganese layers in Co/Mn superlattices

Eur. Phys. J. B 19, 225-239 (2001)

17. F. BENSMINA, A. DINIA, C. MENY, P. PAAISSSJD, D. MULLER

Annealing effect on structural and magn tic aroperties of $\mathrm{Ta} / \mathrm{Cu} / \mathrm{FeMn} / \mathrm{Co} / \mathrm{FeMn}$ thin film structures

Eur. Phys. J. AP, 11, 97-101 (2000)

18. J.L. BUBENDORFF, C. MENY, E. EAI REPAIRE, P. PANISSOD, J.P. BUCHER

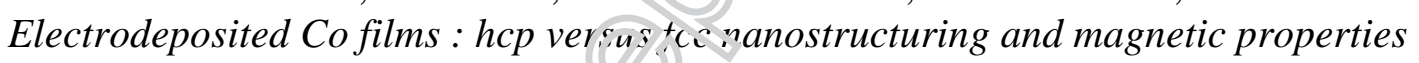

Eur. Phys. J. B 17, 635-643 (200),

19. S. COLIS , A. DINIA, C. MENV,? PANISSOD, C. ULHAQ-BOUILLET,

G. SCHMERBER

Magnetic, transport, and structur. $\mathrm{Fe} / \mathrm{Co} / \mathrm{Cu} /[\mathrm{Co} / \mathrm{Ir}]$ multilayers prepared by ion-beam sputtering

Phys. Rev. B 62, 11709-11718 (2000)

20. J.L. BUBENDORFF, E. BEAUREPAIRE, C. MÉNY, J.P. BUCHER

Overpotential driven perpendicular magnetization of electrodeposited ultrathin cobalt films

J. Appl. Phys. 83, 7043-7045 (1998)

21. H. EL FANITY, K. RAHMOUNI, M. BOUANANI, A. DINIA, G. SCHMERBER,

C. MENY, P. PANISSOD, A. CZIRAKI, F. CHERKAOUI, A. BERRADA

Structural properties of electrodeposited Co/Cu multilayers

Thin Solid Films 318, 227-230 (1998)

22. S. ZOLL, A. DINIA, J.P. JAY, C. MENY, G. Z. PAN, A. MICHEL,

L. EL CHAHAL, V. PIERRON-BOHNES, P. PANISSOD, H. A. M. VAN DEN BERG

Influence of the growth technique on the coupling and magnetoresistance of Co/Ru sandwiches

Phys. Rev. B 57, 4842-4848(1998)

23. M. WOJCIK, J.P. JAY, P. PANISSOD, J. DEKOSTER, G. LANGOUCHE

New phases and chemical short range order in co-deposited CoFe thin films with bcc structure: an NMR study

Z. Phys. B 103, 5-12 (1997) 
24. J.L. BUBENDORFF, E. BEAUREPAIRE, C. MENY, P. PANISSOD, J.P. BUCHER Perpendicular magnetization in ultrathin electrodeposited cobalt films Phys. Rev. B 56, R7120-R7123 (1997)

25. J.P. JAY, E. JEDRYKA, M. WOJCIK, J. DEKOSTER, G. LANGOUCHE P. PANISSOD

On the stability of bcc Co in Co/Fe superlattices an NMR and XRD study

Z. Phys. B 101, 329-337 (1997)

26. N. PERSAT, A. DINIA, J.P. JAY, C. MENY, P. PANISSOD

Structural properties and oscillatory magnetoresistance of $\mathrm{Co}(\mathrm{hcp}) / \mathrm{Cu}$ sandwiches

J. Magn. Magn. Mater. 164, 37-42 (1996)

27. N. PERSAT, A. DINIA, J.P. JAY, C. MENY, P. PANISSOD

Structure and oscillatory magnetoresistance of Co(hcp)/Cu sandwiches

Thin Solid Films 275, 115-118 (1996)

28. S. ZOLL, H. A. M. VAN DEN BERG, J.-P. JAY, H. J. ELMERS, C. MENY,

P. PANISSOD, D. STOEFFLER, A. DINIA, K. OUNADJELA

Coupling mechanism in Co/Ru sandwiches with thin spacers

J. Magn. Magn. Mater. 156, 231-232 (1996)

29. N. PERSAT, A. DINIA, J.P. JAY, C. MENY, P. PANISSOD

Structure and giant magnetoresistance in $\mathrm{Co} / \mathrm{Cu}$ sandwiches with thin Ag layers at the interfaces

J. Magn. Magn. Mater. 156, 335-336 (1996)

30. PANISSOD, J.P. JAY, C. MENY, M. WOJC IN IEDRYKA

NMR analysis of buried metallic interfac-s

$10^{\text {th }}$ International Conference on Hyperfirc interactions, Louvain, Belgique, 28 Août-1

Septembre 1995

Hyperfine Interact. 97-98, 75-98 (190,5)

31. P. PANISSOD, C. MENY, J.P. JA WOJCIK, E. JEDRYKA

Structure des multicouches métali i es de leurs interfaces vues par RMN

$39^{\text {ème }}$ Colloque de Métallurgie. Saciay, 25-27 Juin 1996

J. Phys. IV (Paris) Suppl. Co !. 7 ó. C7_89-106 (1996)

32. P. PANISSOD, J.P. JAY, C. MEi Y, M. WOJCIK, E. JEDRYKA

NMR studies of bulk and interface structure in Co based multilayers

Symposium on Magnetic Ultrathin Films, Multilayers and Surfaces, San Francisco, USA, 17-20 Avril 1995

Mater. Res. Soc. Symp. Proc. 384, 61-72 (1995)

33. J. DEKOSTER, E. JEDRYKA, C. MENY, G. LANGOUCHE

Epilayer induced structural transition to bcc Co during epitaxial growth of Co/Fe superlattices

Europh. Lett. 22, 433-438 (1993)

34. Y. HENRY, C. MENY, A. DINIA, P. PANISSOD

Structural and magnetic properties of semiepitaxial Co/Cr multilayers

Phys. Rev. B 47 15037-15045 (1993)

35. C. MENY, P. PANISSOD, P. HUMBERT, J.P. NOZIÈRES, V.S. SPERIOSU, B.A. GURNEY, R. ZEHRINGER

Structural study of $\mathrm{Cu} / \mathrm{Co} / \mathrm{NiFe} / \mathrm{FeMn}$ spin valves by nuclear magnetic resonance J. Magn. Magn. Mater. 121, 406-408 (1993)

36. T. VALET, P. GALTIER, J.C. JACQUET, C. MENY, P. PANISSOD

Correlation between giant magnetoresistance and the microstructure of $\left[\mathrm{Ni} 80 \mathrm{Fe}_{20} / \mathrm{Cu} / \mathrm{Co}\right]$ multilayers

J. Magn. Magn. Mater. 121, 402-405 (1993) 
37. J. DEKOSTER, E. JEDRYKA, C. MENY, G. LANGOUCHE Epitaxial growth of bcc Co/Fe superlattices J. Magn. Magn. Mater. 121, 69-72 (1993)

38. P. PANISSOD, C. MENY NMR investigation of the nanostructure of .../Cu/Co/Cu... layers J. Magn. Magn. Mater.126, 16-18 (1993)

39. C. MENY, J.P. JAY, P. PANISSOD, P. HUMBERT, V.S. SPERIOSU, H. LEFAKIS, J.P. NOZIÈRES, B.A. GURNEY

Annealed $\mathrm{Cu} / \mathrm{Co} / \mathrm{Cu} / \mathrm{NiFe} / \mathrm{FeMn}$ spin valves : nanostructure and magnetism Mat. Res. Soc. Symp. Proc. 313, 289 (1993)

40. C. MENY, P. PANISSOD, R. LOLOEE

Structural study of cobalt-copper multilayers by NMR Phys. Rev. B 45, 12269-12277 (1992)

41. N. YAACOUB, C. MENY, C. ULHAQ-BOUILLET, M. ACOSTA, P. PANISSOD Short period magnetic coupling oscillations in Co/Si multilayers: on the role of crystallization and interface quality

Phys. Rev.B, 75, 174402,( 2007)

42. N. YAACOUB, C. MENY, O. BENGONE, P. PANISSOD

Reduced interfacial mixing and exchange coupling in Co/Si multilayers

J. Magn. Magn. Mater. 316, e980-e98 (2007)

43. N. YAACOUB, C. MENY, O. BENGONE, P. YANISSOD

Short period magnetic coupling oscillation in Co/Si multilayers: theory versus experiment

Phys. Rev. Lett. 97, 257206 (2006)

44. M. VÉLEZ, C. MENY, S.M. VAi YIDARES, J. DIAZ, R. MORALES, L.M. ALVAREZ-PRADO, P. PANIS ;OD, J.M. ALAMEDA

Amorphous to polycrystalline transfiijs in Cox $_{x} i_{1-x}$ alloy thin films

Eur. Phys. J. B 41, 517-524 (200 f)

45. Y.Y. YUAN, K. LE GUEN, J.M ANDRE, C. MENY, C. ULHAQ, A. GALTAYRIES, J.T. ZHU, Z.S. WANG, P. JC.INN, RD,

Interface observation of heat-treared $\mathrm{Co} / \mathrm{Mo} 2 \mathrm{C}$ multilayers

Appl. Surf. Sci. 331, 8-16 (2015)

46. Y.Y. YUAN, K. LE GUEN, J. M. ANDRE, Z.S. WANG, J.T. ZHU, C. MENY, P. JONNARD

Optical and structural characterization of the $\mathrm{Co} / \mathrm{Mo} 2 \mathrm{C} / \mathrm{Y}$ system Appl. Surf. Sci. 315, 499-505 (2014)

47. K. LE GUEN, M.-H. HU, J.-M. ANDRÉ, S. K. ZHOU, H. CH. LI, J. T. ZHU, Z. S. WANG, C. MENY, A. GALTAYRIES, P. JONNARD

Observation of an asymmetrical effect when introducing $\mathrm{Zr}$ in $\mathrm{Mg} / \mathrm{Co}$ multilayers Appl. Phys. Let. 98, 251909 (2011)

48. K. LE GUEN, M.H. HU, J.M. ANDRE, P JONNARD, P.S.K. ZHOU, H. LI, , J.T. ZHU, Z.S. WANG, C. MENY,

Development and characterization of Co/Mg periodic multilayers for EUV applications J. Phys. Chem. C, 114, 6484-6490 (2010)

49. G. AVEDISSIAN, J. ARABSKI, J. A. WYTKO, J. WEISS, C. MENY

Revealing the morphology and the magnetic properties of single buried cobalt-ZnTPP hybrid interfaces by FNR spectroscopy

Phys. Rev. B 102, 184114 (2020)

50. G. AVEDISSIAN, J. ARABSKI, J. A. WYTKO, J. WEISS, C. MENY 
Probing the Growth of Organic Molecular Films Embedded between Cobalt and Iron Electrodes: Ferromagnetic Nuclear Resonance Approach

Adv. Funct. Mater. 30, 2005605 (2020)

51. F. GODEL, C. MENY, B. DOUDIN, H. MAJJAD, J.F. DAYEN, D. HALLEY

Epitaxial ferromagnetic single clusters and smooth continuous layers on large area MgO/CVD graphene substrates

Materials Research Express, 5, 025606, (2018)

52. E. DUJARDIN, C. MENY, P. PANISSOD, J.P. KINTZINGER, N. YAO,

T.W. EBBESEN

Interstitial metallic residues in purified single shell carbon nanotubes

Solid State Comm. 114, 543-546 (2000)

53. C. GARNERO, M. LEPESANT, C GARCIA-MARCELOT, Y. SHIN, C. MENY,

P. FUGER, B. WAROT-FONROSE, R. ARENAL, G. VIAU, K. SOULANTICA, P. FAU,

P. POVEDA, L.M. LACROIX, B. CHAUDRET

Chemical ordering in bimetallic FeCo nanoparticles: from a direct chemical synthesis to application as efficient high-frequency magnetic material

Nanoletters 19, 1379-1386 (2019)

54. Y.F. LIU, J.G. LUO, Y. SHIN, S. MOLDOVAN, O. ERSEN, A. HÉBRAUD, G. SCHLATTER, C. PHAM-HUU, C. MENY

Sampling the structure and chemical order in assemblies of ferromagnetic nanoparticles by Nuclear Magnetic Resonance

Nature Commun. 7:11532 doi: 10.1038/rims i 1532 (2016)

55. Y.F. LIU, I. FLOREA, O. ERSEN, C. PFi div tiUU, C. MENY

Silicon carbide coated with $\mathrm{TiO} 2$ with entanced cobalt active phase dispersion for Fischer-Tropsch synthesis

ChemCommun, 51, 145-148 (2015

56. Y.F. LIU, J. LUO, M. GIRLEANJU C ERSEN, C. PHAM-HUU, C. MENY

Efficient Hierarchically Structurea Composites Containing Cobalt Catalyst for Clean Synthetic Fuel Production frcin Fishcer-Tropsch Synthesis

J. Cat. 318, 179-192 (2014)

57. Y.F. LIU, O.ERSEN, C. MENY, F. LUCK, C. PHAM-HUU

Fischer-Tropsch Reaction on a Thermally Conductive and Reusable Silicon Carbide Support

ChemSusChem 7, 1218-1239 (23014)

58. Y. LIU, B.DE TYMOWSKI, F. VIGNERON, I. FLOREA, O.ERSEN, C. MENY, P. NGUYEN, C. PHAM, F. LUCK, C. PHAM-HUU

Titania-decorated silicon carbide-containing Cobalt catalyst for Fischer-Tropsch synthesis

ACS Catal. 3, 393-404 (2013)

59. B. DE TYMOWSKI,Y. LIU, C. MENY, C. LEFÈVRE, D. BEGIN,P. NGUYEN, C. PHAM, D. EDOUARD, F. LUCK, C. PHAM-HUU

$\mathrm{Co}-\mathrm{Ru} / \mathrm{SiC}$ impregnated with ethanol as an effective catalyst for the Fischer-Tropsch synthesis

Appl. Catalysis A. 31, 418-420 (2012)

60. G. POURROY, A. VALLES-MINGUEZ, I.S. JURCA, C. MENY, P. PANISSOD

Synthesis and properties of $\mathrm{Fe}^{0}-\mathrm{Co}^{0} / \mathrm{Co}$-magnetite composites under hydrothermal conditions

J. Alloys Comp. 333, 296-301 (2002)

61. J.P. JAY, I.S. JURCA, G. POURROY, N. VIART, C. MENY, P. PANISSOD 
${ }^{59}$ Co NMR study in Fe-Co alloys/Co magnetite composites

Solid State Sciences 3, 301-308 (2001)

62. M. MALINOWSKA, M. WOJCIK, S. NADOLSKI, E. JEDRYKA, C. MENY, P. PANISSOD, M. KNOBELL, A. D. C. VIEGAS, J. E. SCHMIDT

Identification of magnetic phases in granular Co $10 \mathrm{Cu}$ (uo alloy using ${ }^{59} \mathrm{Co}$ NMR method J. Magn. Magn. Mater. 198-199, 599-601 (1999)

63. C. MENY

Etude de multicouches magnétiques par RMN: Méthodologie et application aux systems $\mathrm{Co} / \mathrm{Cu}, \mathrm{Co} / \mathrm{Cr}, \mathrm{Co} / \mathrm{Ru}$ et $\mathrm{Co} / \mathrm{Fe}$

PhD manuscript, Université Louis Pasteur, Strasbourg, France (1994)

64. J.P. JAY

Etude par resonance magnétique nucléaire de l'ordre à courte distance dans le système Cobalt/Fer: De l'alliage massif à la multicouche

PhD manuscript, Université Louis Pasteur, Strasbourg, France (1995)

65. J. F. M. L. MARIANO, M. ROGALSKI, O. POSTOLACHE,

A simulation based comparative study of two broadband probes for NMR of magnetically ordered materials

2011 Fifth International Conference on Sensing Technology, Palmerston North, 161-164 (2011)

66. A.M. PORTIS, A.C. GOSSARD

Nuclear resonance in ferromagnetic Co

J. Appl. Phys. Suppl. 31 205S (1960)

67. H. AKAI, M. AKAI, S. BLIIGEL, B. DRI ILER, H. EBERT, K. TERAKURA, R. ZELLER P. DEDDERICHS

Theory of Hyperfine Interactions in Metais

Progr. Theor. Phys. Suppl. 101, l 1 (1990

68. M.B. STEARNS

Spin echo and free induction deccin wements in pure Fe and Fe rich ferromagnetic alloys: Domain wall dynamics

Phys. Rev. 162, 496, (1967)

69. E.T. JAYNES

Matrix treatment of nuclear induction

Phys. Rev. 98, 1099, (1955)

70. S. SHTRIKMAN, E.P. WOHLFARTH

The theory of the Vogel-Fulcher law of spin glasses

Phys. Lett. A 85, 467-470 (1981) 


\section{Table}

$\begin{array}{lllllllllllllllll}\text { Sequence } & 1 & 2 & 3 & 4 & 5 & 6 & 7 & 8 & 9 & 10 & 11 & 12 & 13 & 14 & 15 & 16\end{array}$

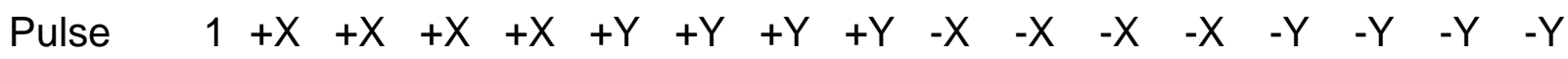
along

$\begin{array}{llllllllllllllllllllllllllll}\text { Pulse } & 2 & + & + & + & -Y & -X & +X & +Y & -Y & -X & +X & +Y & -Y & -X & +X & + & -Y & -X\end{array}$ along

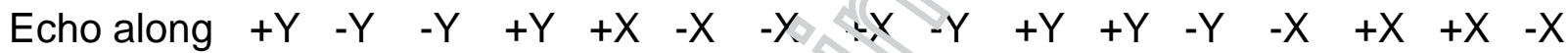

Table 1. Phase permutation of the two prise sequence and resulting spin echo direction. 


\section{Figures}

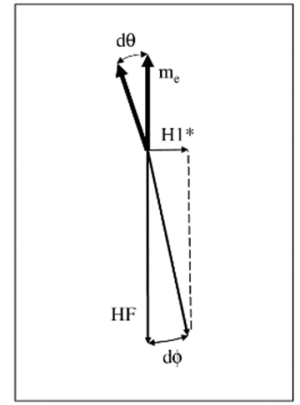

Figure 1. Schematic drawing of the enhancement factor effect. Under the RF H1 the electronic magnetisation $m_{e}$ rotates by an angle $d \theta$ resulting in the rotation of the HF by an angle $d \phi$. The perpendicular component of the motion of the $\mathrm{HF}$ is the RF field $\mathrm{H} 1 *$ experienced by the Nuclei.

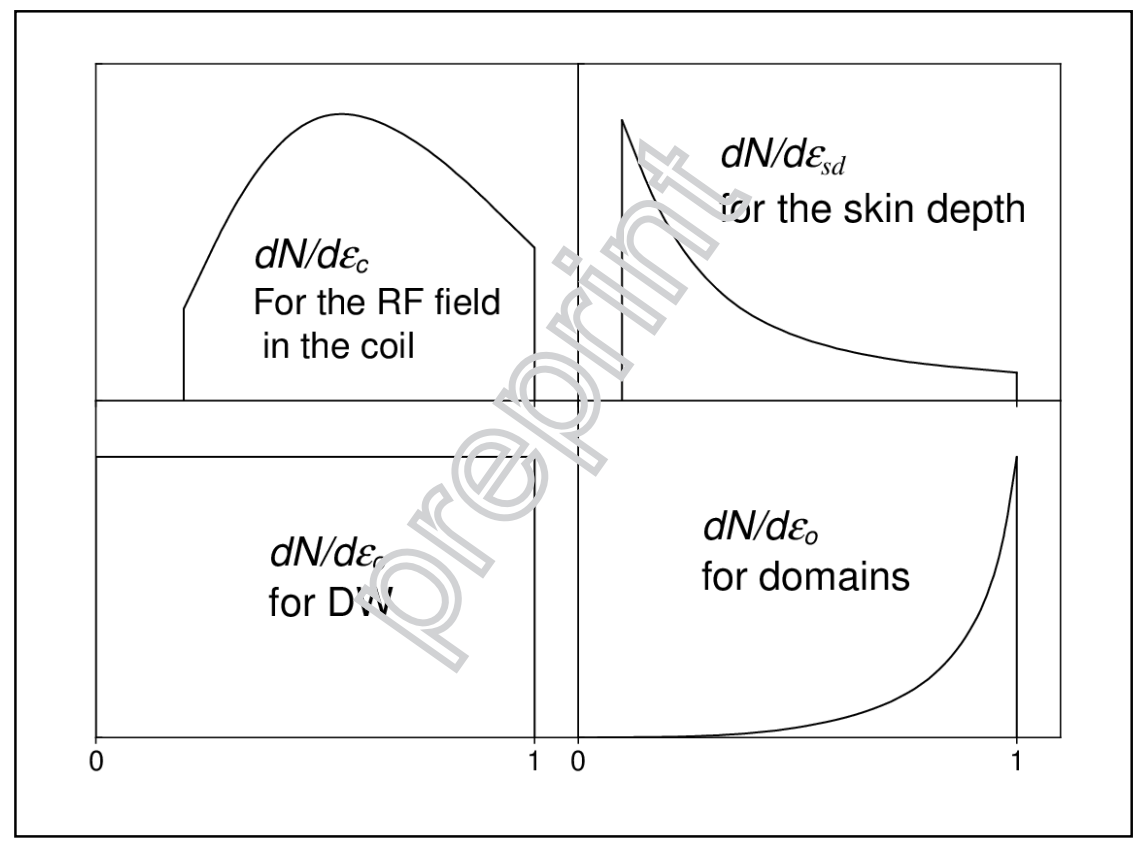

Figure 2. Typical shapes of distributions of $\varepsilon_{i}$. These distributions are difficult to compute and will vary in shape from sample to sample. However, their qualitative behaviours can be inferred. For the RF field inside the coil. It is maximum in the middle of the coil and it will decrease towards its edges and of axis of the coil. Most of the nuclei will therefore experience a field somewhat smaller than the maximum possible RF field. In bulk metallic samples a limited number of the nuclei at the surface will experience the maximum RF field. Towards the centre of the sample more and more nuclei will experience a smaller and smaller field. For nuclei in magnetic domain walls a wide spread of RF field will result from the DW orientation. Finally, the distribution of the domain orientations will be dominated by the domain oriented at $90^{\circ}$ to the RF field. 


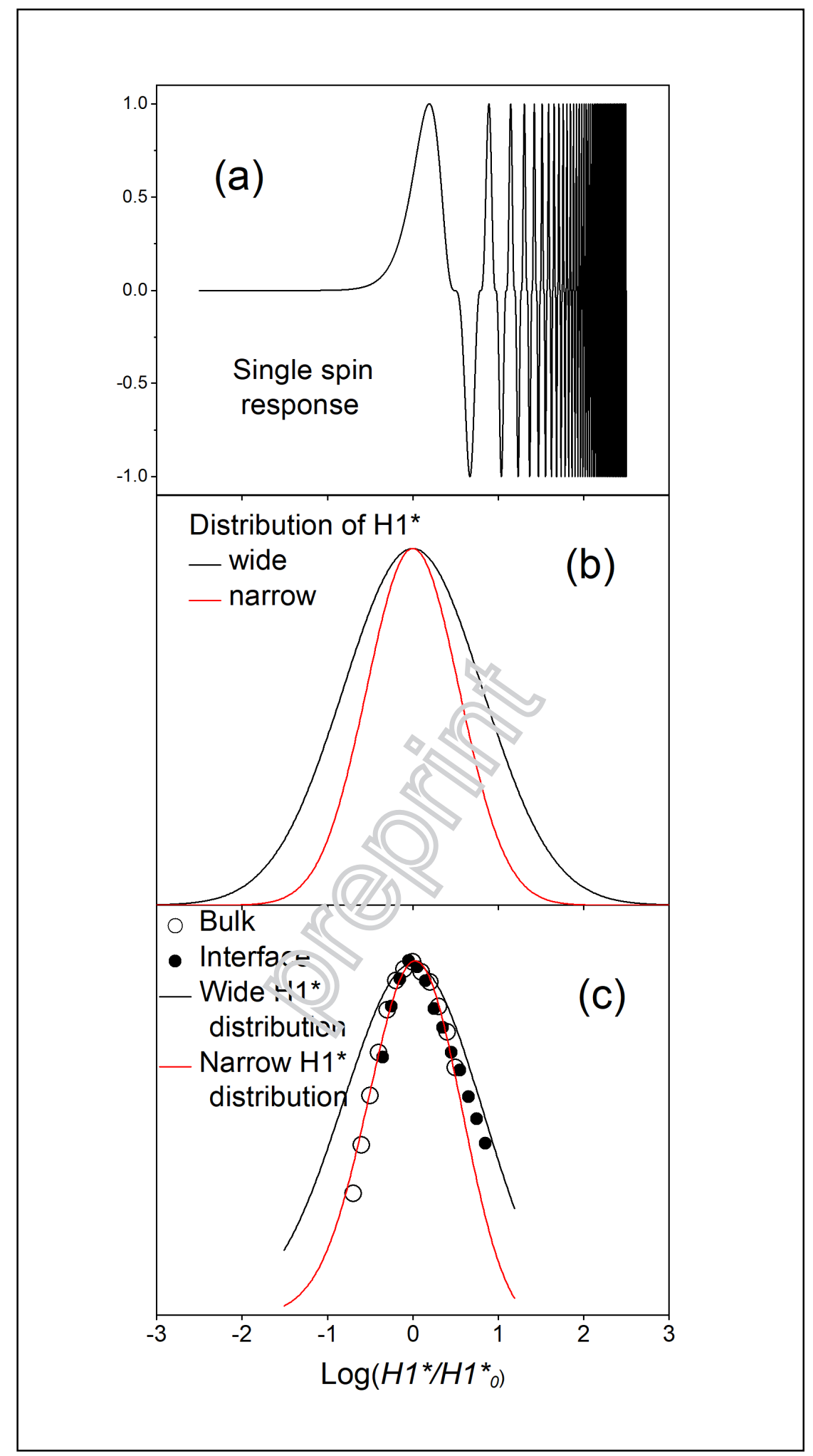

Figure 3. (c): Convolution product of the spin response (a) with the distribution of $H l^{*}$ (b). Circles are experimental data obtained on a Co thin film. The convolution product has a log normal (Gaussian in log scale) shape close to its maximum. 


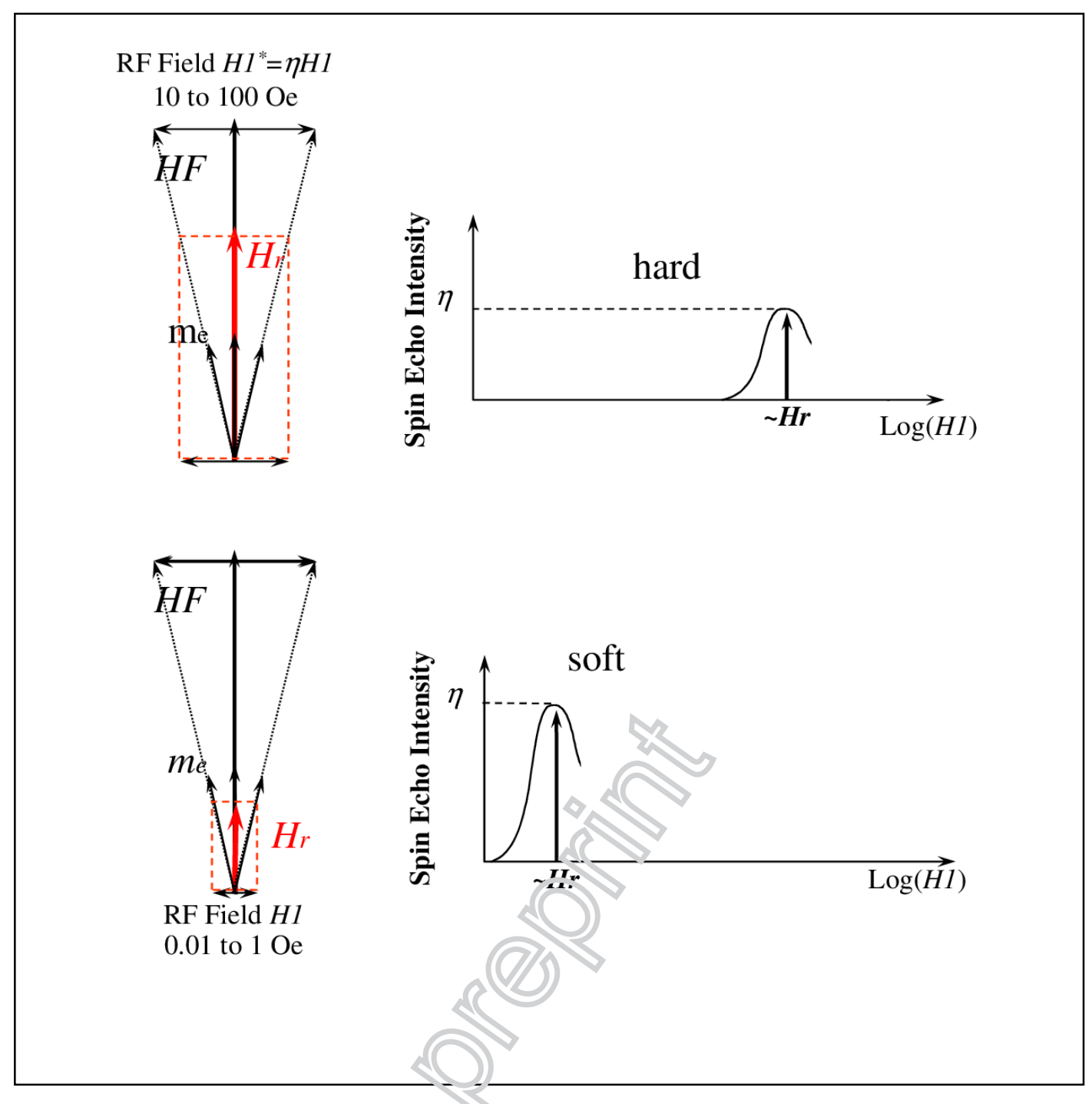

Figure 4. Restoring field (Hr) description of the enhancement faction. HF: hyperfine field; $\mathrm{m}_{\mathrm{e}}$ : electronic magnetisation, H1 applied RF field. For simplicity the HF has been drawn parallel to the $m_{e}$ direction while usually it is pointing in the opposite direction. 


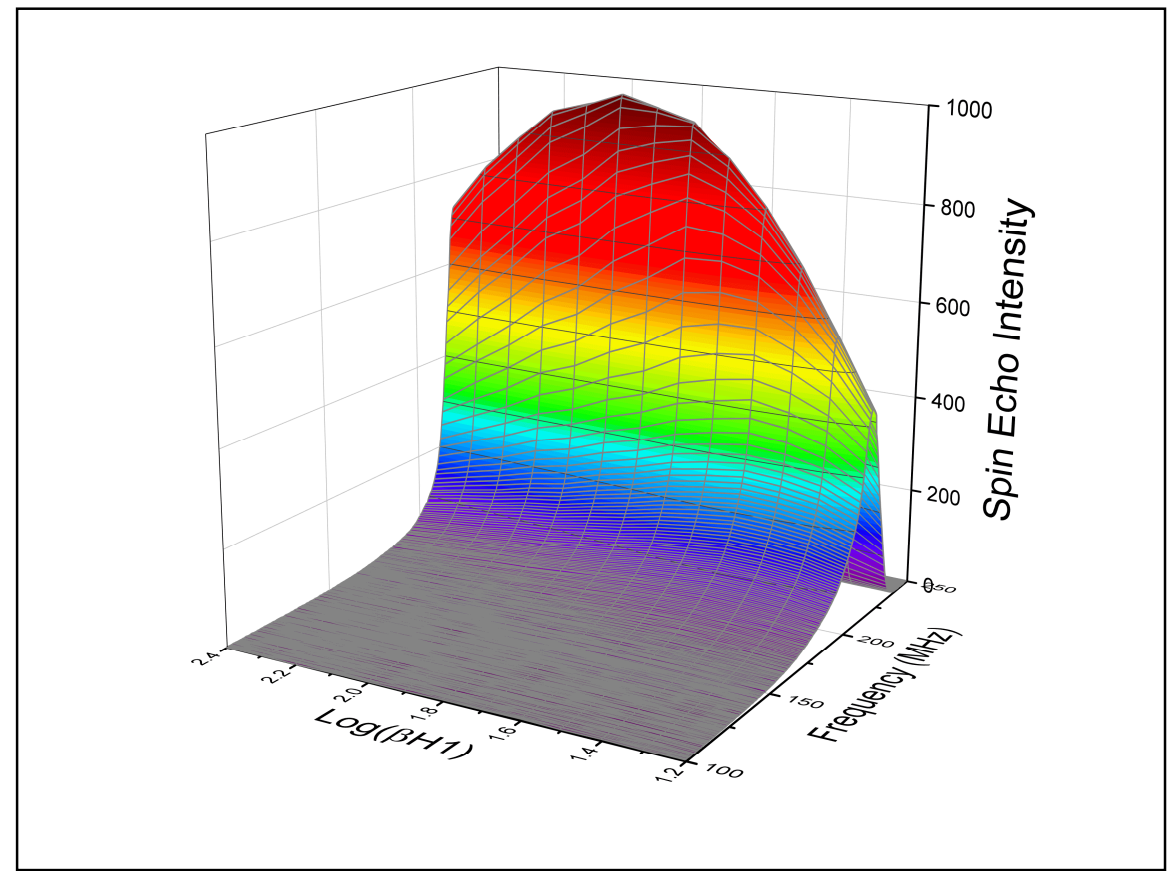

Figure 5. 3D plot of the spin echo intensity versus the asplied radiofrequency field $H I$ frequency and amplitude: $H 1$ 2D mapping. The shift witi ire frequency of the optimum $H 1$ amplitude value required for reaching the maximan! GNR signal can be easily observed.

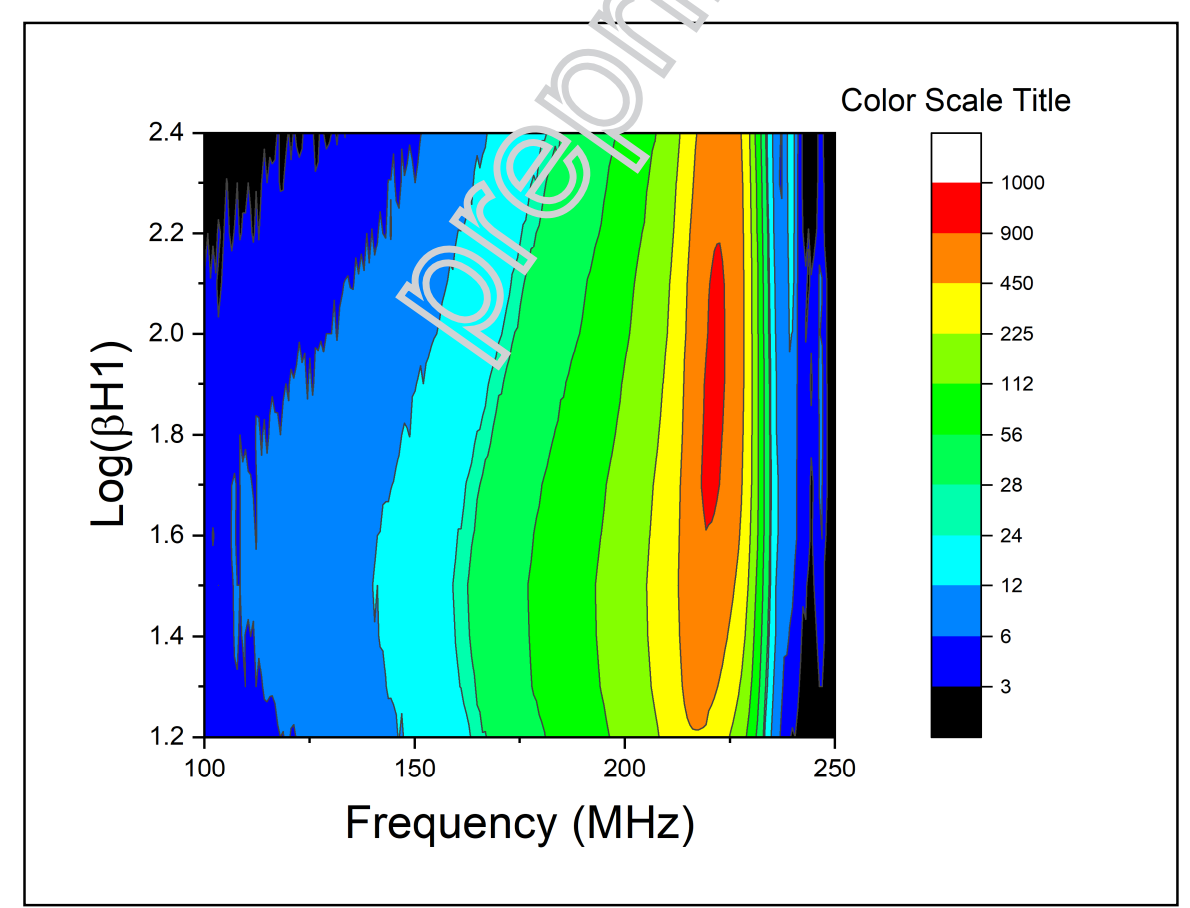

Figure 6. Contour plot of the spin echo intensity versus the applied radiofrequency field $H 1$ frequency and amplitude for the same sample as in Figure 5. 


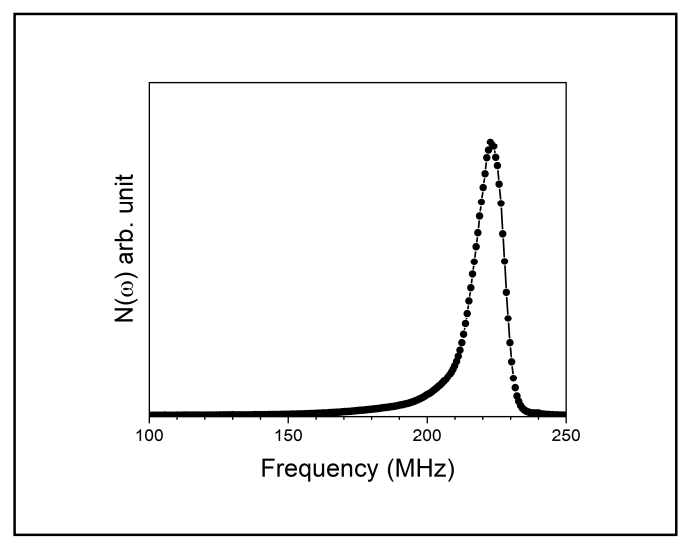

Figure 7. Example of true nuclear spin distribution versus resonance frequency for the same sample as in figure 5 to 7.

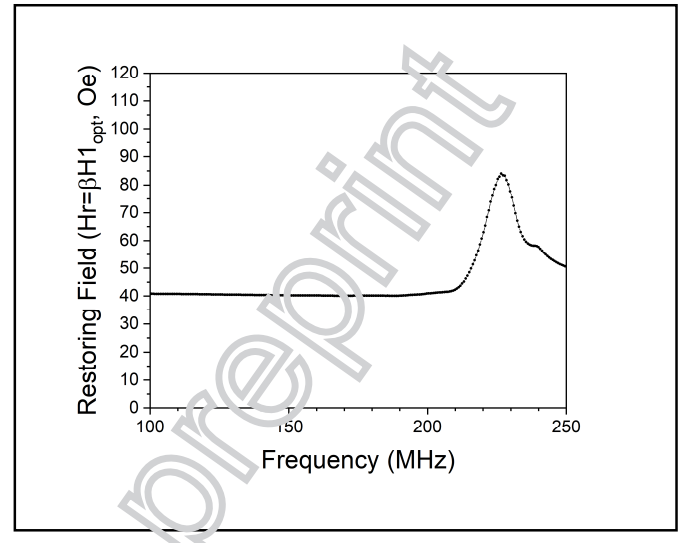

Figure 8. Example of frequency dependent restoring field $\mathrm{Hr}=\beta \mathrm{H} 1_{\text {opt }}$ computed for the same sample as in Figure 5 and 6.

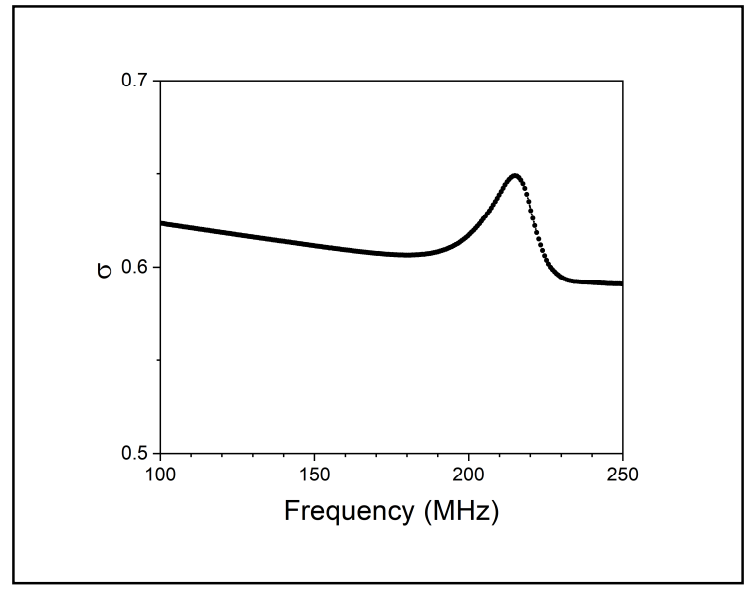

Figure 9. Width of the log normal shape of the FNR signal (same sample as for figure 5 to 8) 


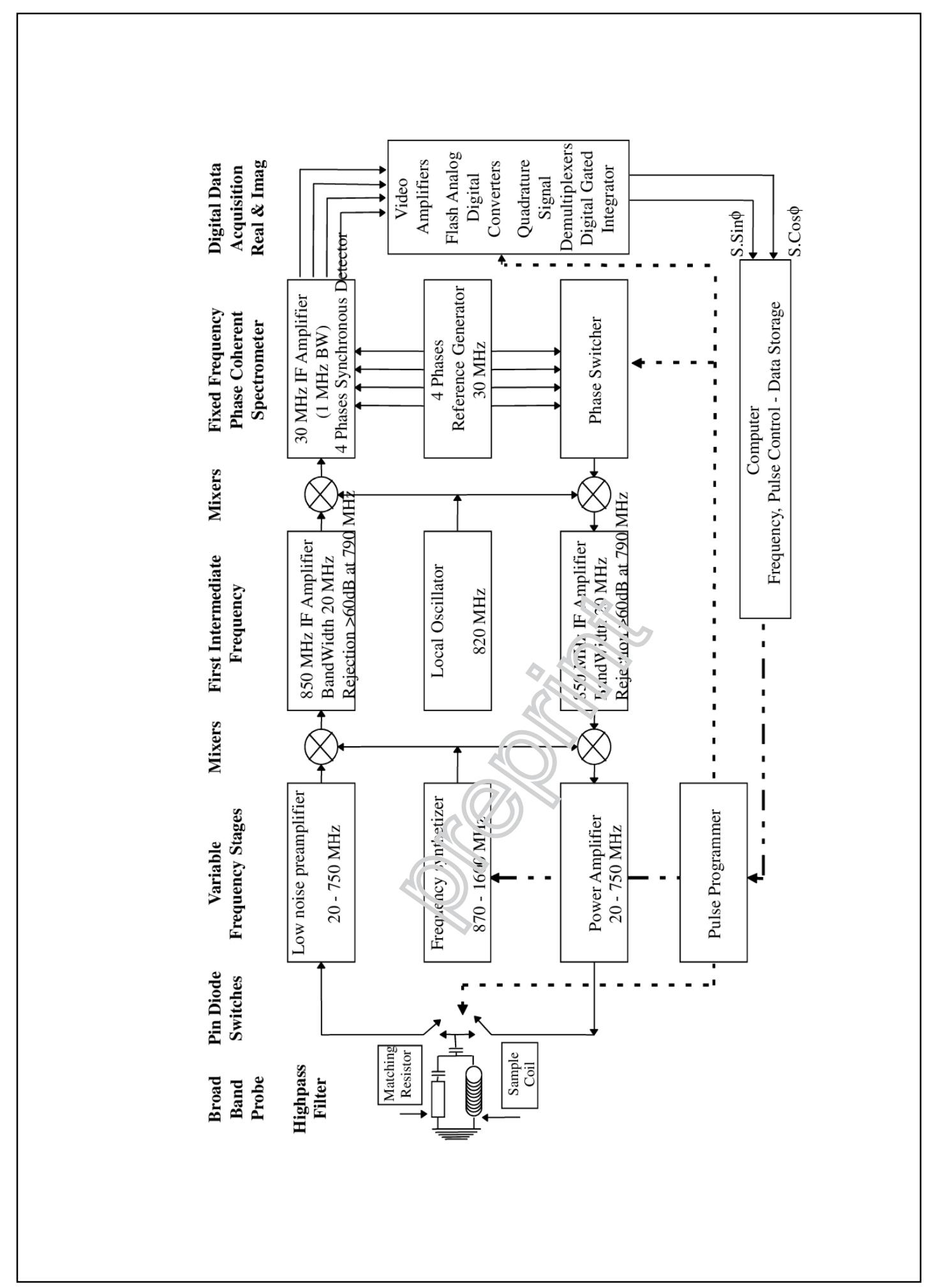

Figure 10. Block diagram of the architecture of our broadband double heterodyne FNR spectrometer with automatic frequency scanning. 


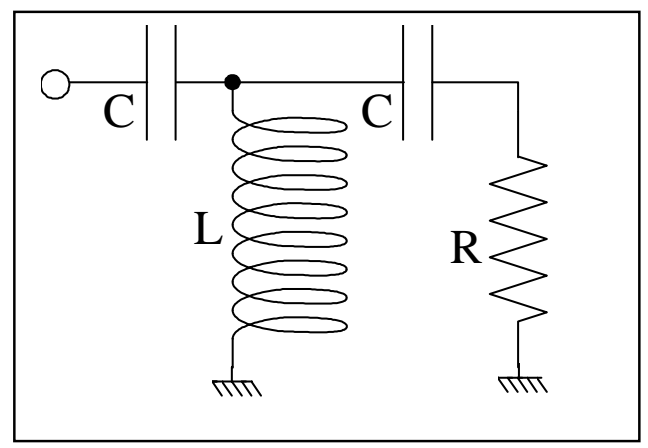

Figure 11. Design of our broad band high pass filter probe.

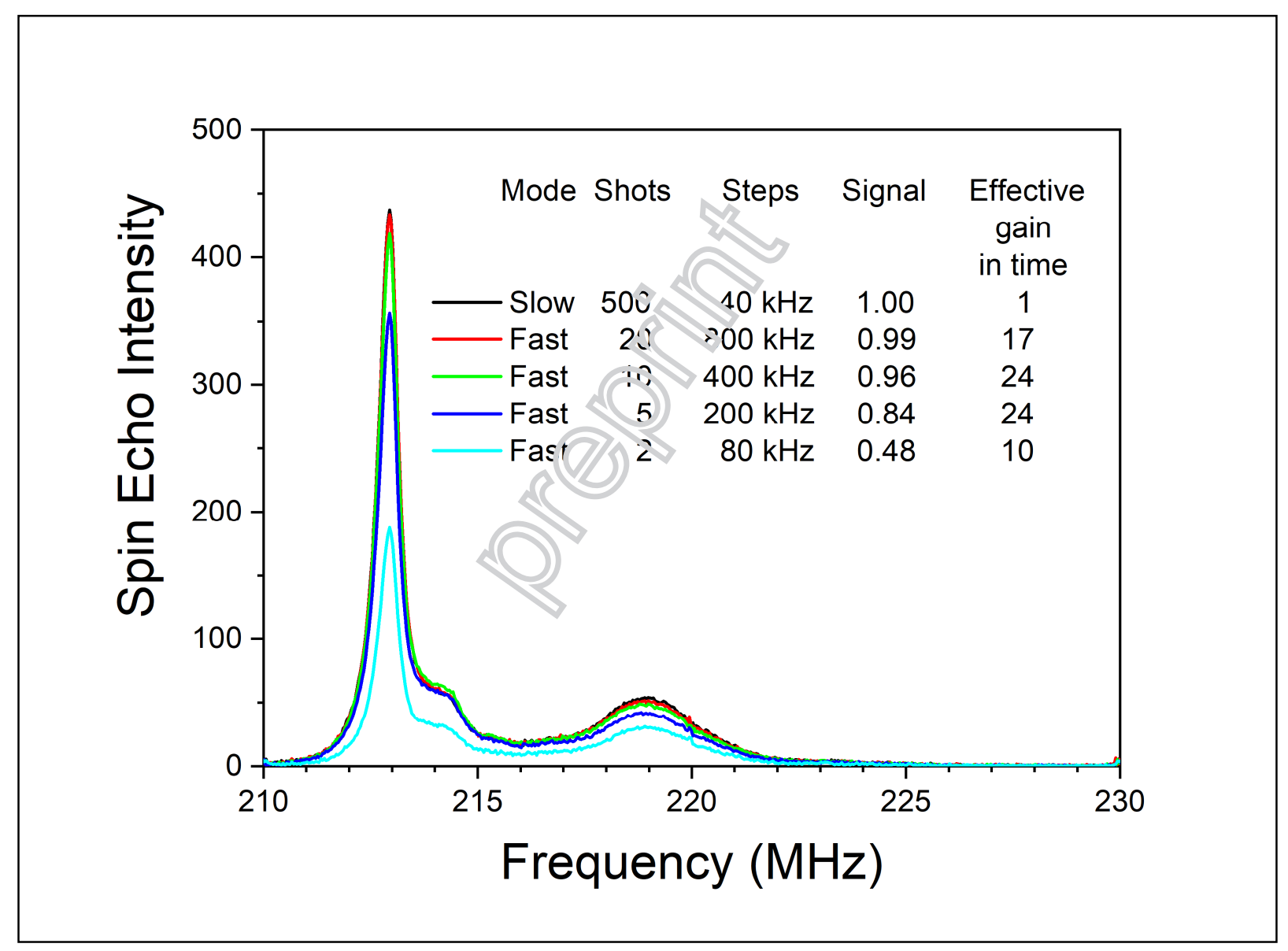

Figure 12. Comparison of FNR spectra obtained by fast scanning modes with the spectrum obtained by slow scanning mode. Measurement is performed at $300 \mathrm{~K}$. If fast scanning mode is slow compared to spin diffusion the FNR signal decreases. The gain in time obtained with the fast accumulation mode is computed by considering the same resulting signal/noise ratio. The gain in time is of the order of 24. Since it is a narrow FNR spectrum this can be considered as a worst case. 


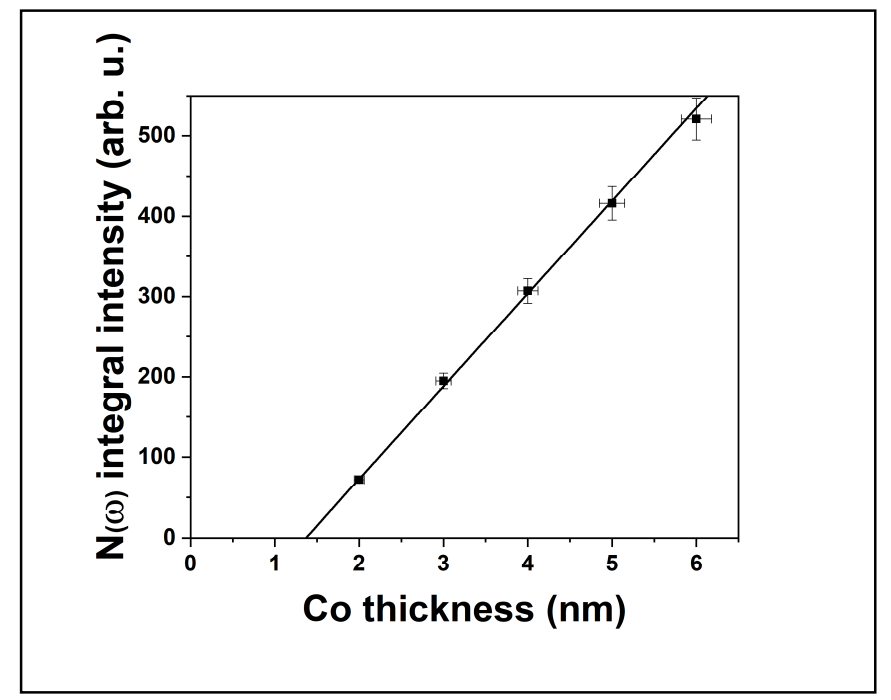

Figure 13. Plot of the $N(\omega)$ integral intensity of ZnTPP/Co/ZnTPP sandwiches versus the deposited Co thickness. Since the spectra are normalized to the samples' surface area the integral intensity increases linearly with the increase of the deposited thickness. The fitted line does not go through zero. It means that some of the Co atoms are ferromagnetic and are therefore not detected by FNR.

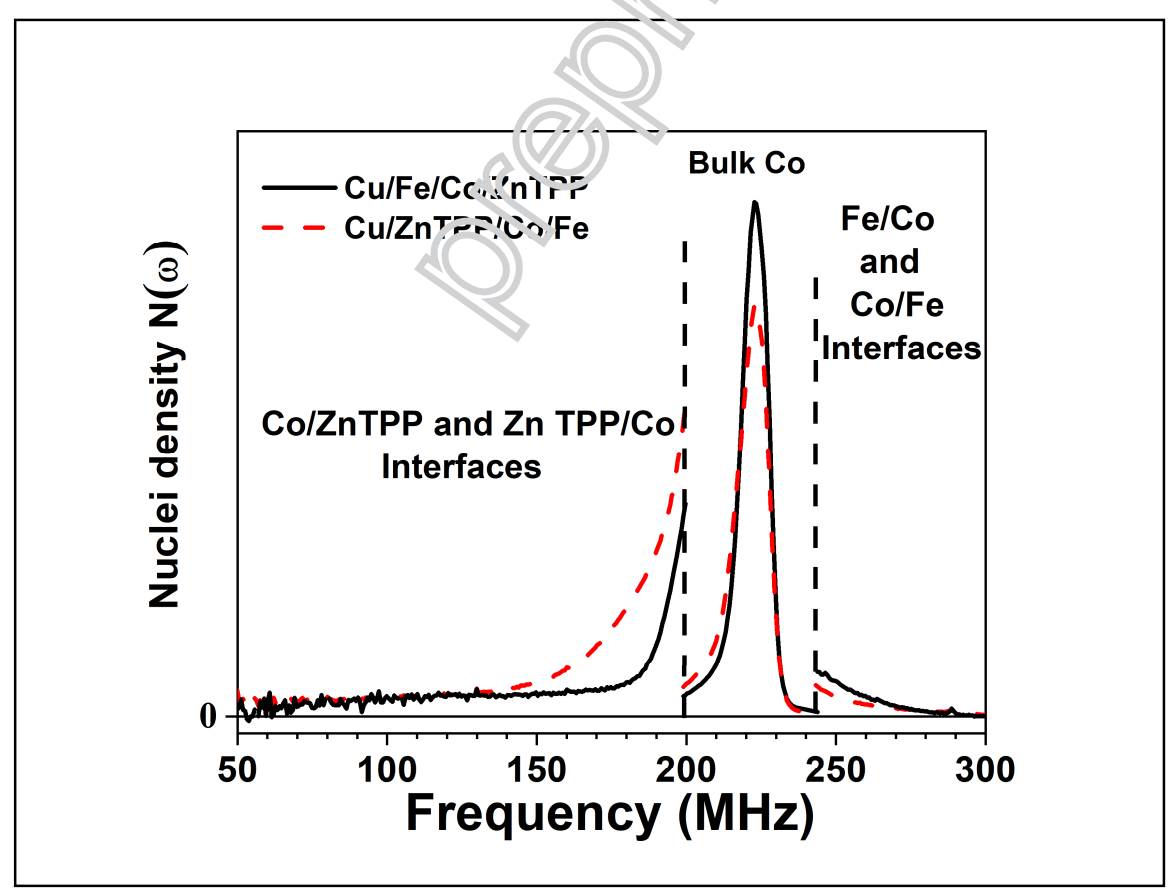

Figure 14. FNR spectra of single buried interfaces. For clarity low frequencies $(<200 \mathrm{MHz})$ and high frequencies $(>245 \mathrm{MHz}$ ) are blown up by a factor of 10 . The Co/ZnTPP and ZnTPP/Co FNR interfacial contributions are observed are low frequency while respectively the Fe/Co and $\mathrm{Co} / \mathrm{Fe}$ interface FNR contributions are observed at high frequency. Interface contributions involve 1 to 4 atomic planes of Co. 


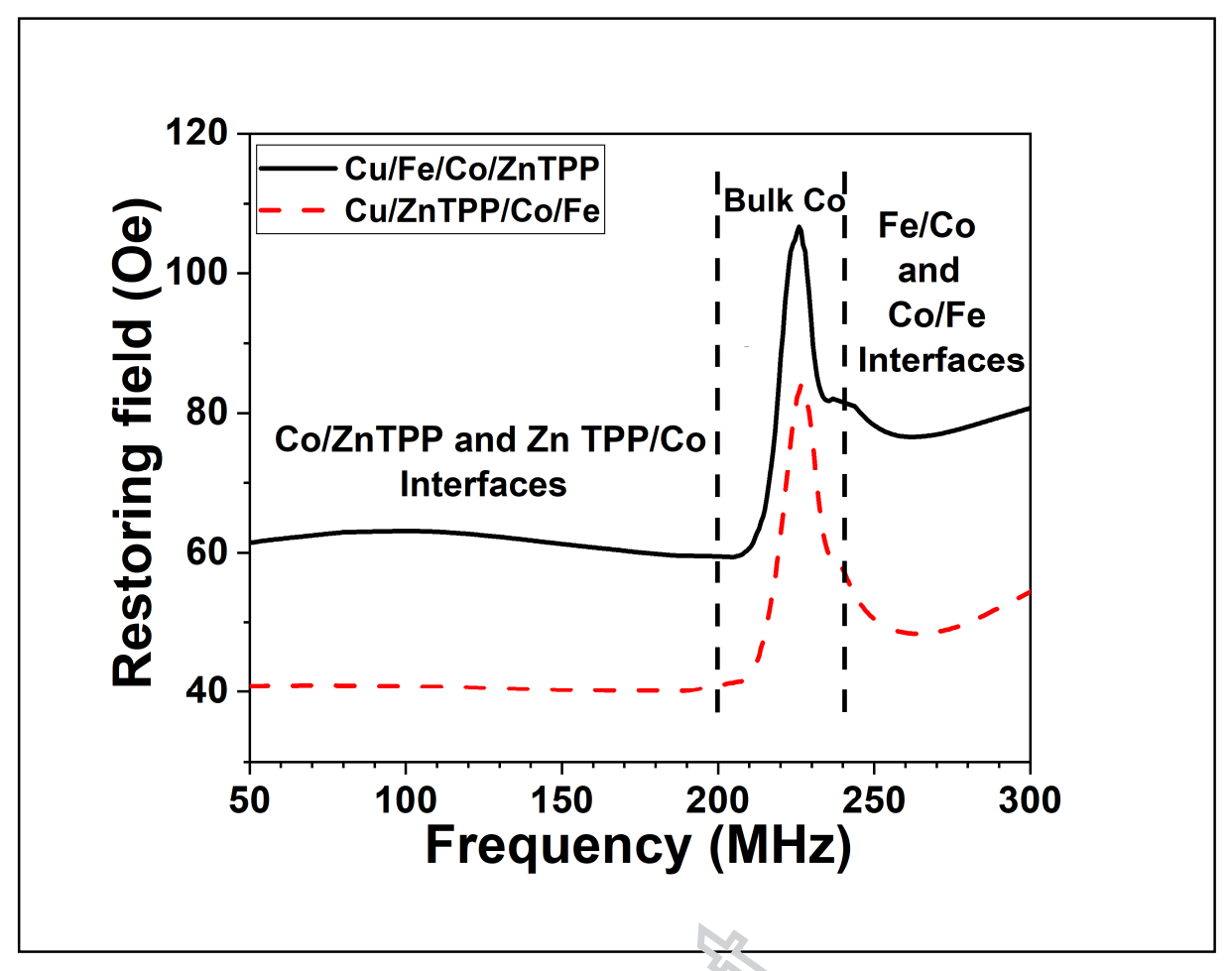

Figure 15. Restoring fields of single buried interfaces. The $\mathrm{Hr}$ can be identified to the local effective anisotropy. Both Co interfaces have a swaile magnetic anisotropy than the bulk part of the layers (Co atoms far from the interfaces) 


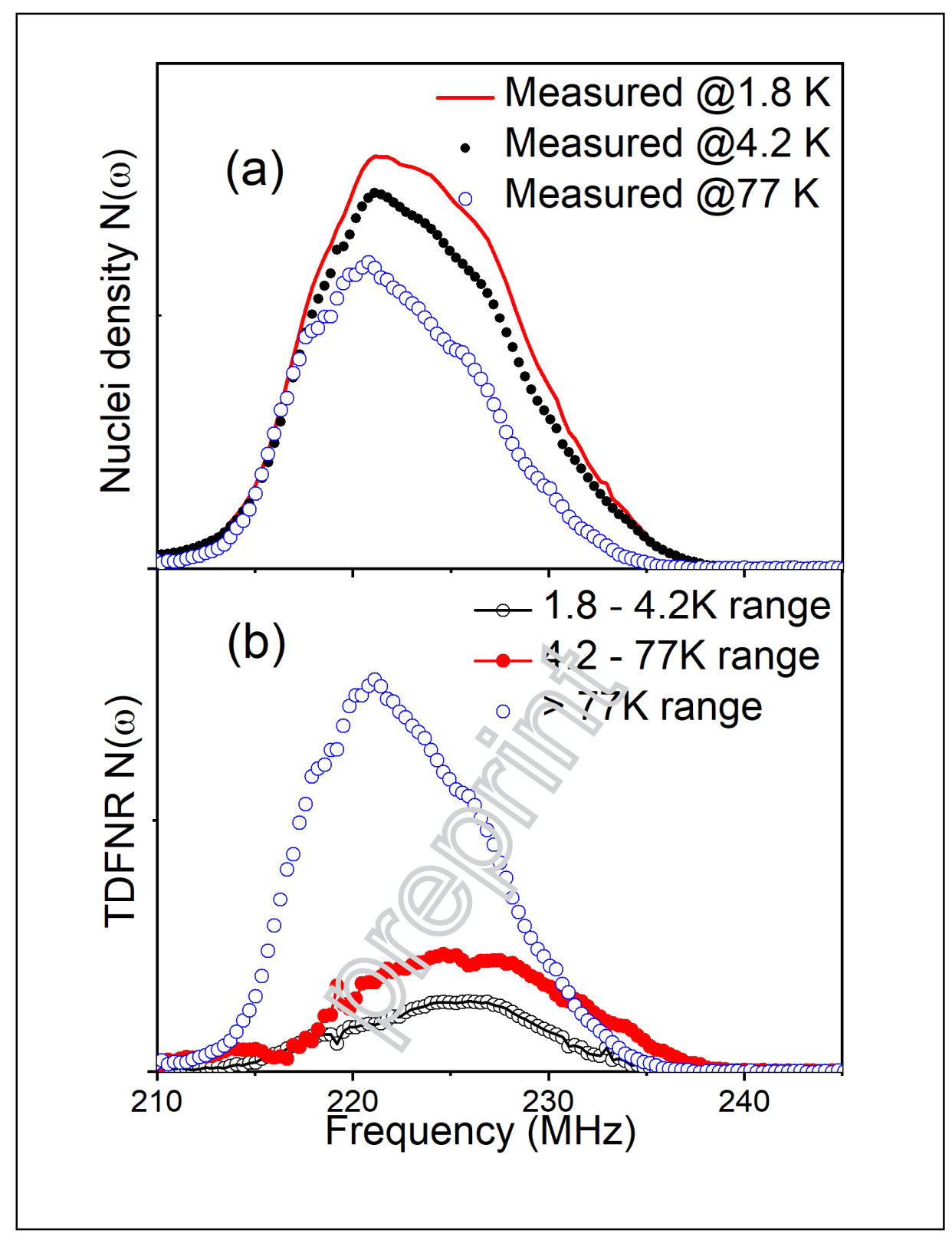

Figure 16. (a), FNR $\mathrm{N}(\omega)$ spectra of an assembly of particles measured at 1.8, 4.2 and $77 \mathrm{~K}$. Increasing the measurement temperature, the smallest particles vanish from the spectra because they become superparamagnetic. (b), TDFNR spectra of the same assembly of particles. The largest particles mostly have an fcc structure while the smallest one mostly have an hcp structure. 


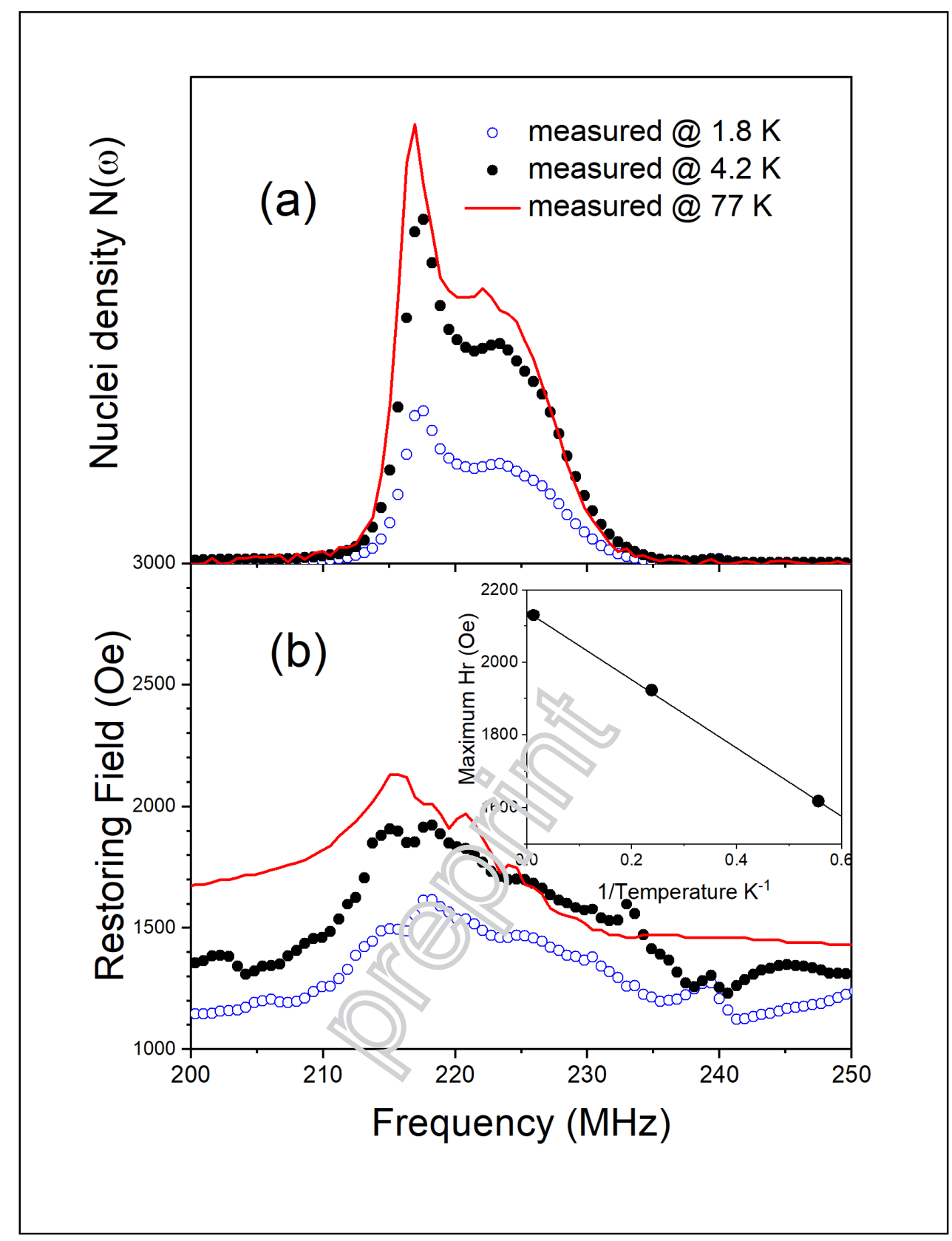

Figure 17. (a) $\mathrm{N}(\omega)$ measured at $77,4.2$ and $1.8 \mathrm{~K}$ for an assembly of particles coupled by dipolar interactions. (b), The restoring field decreases like 1/T (inset). 


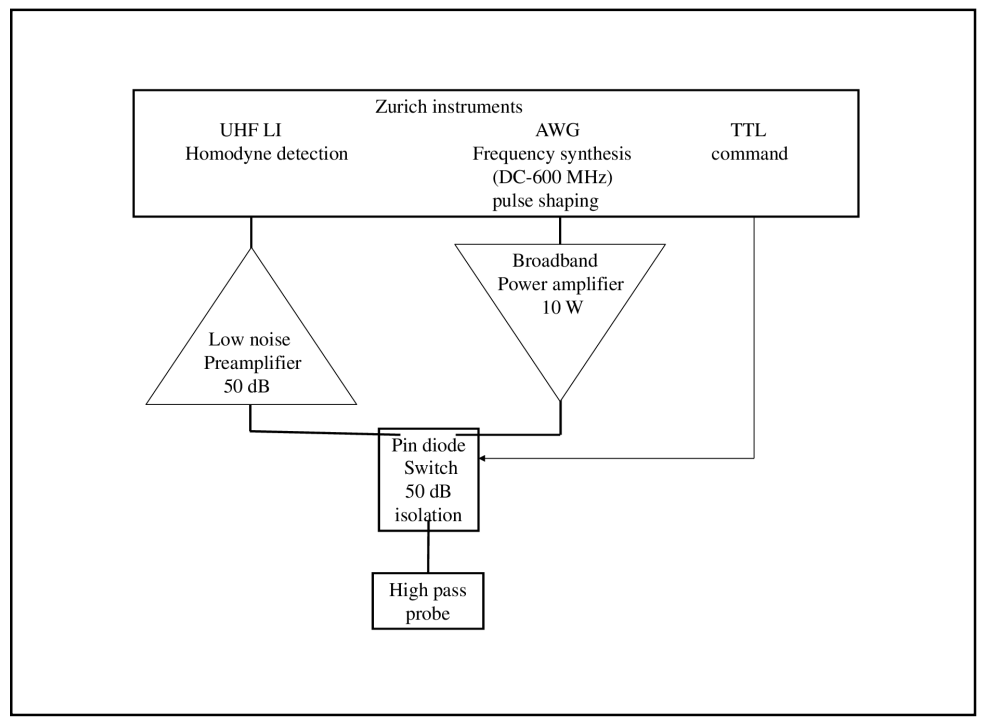

Figure 18. Block diagram for building an FNR spectrometer with automated frequency scanning from on the shelf components. AWG produces the DC to $600 \mathrm{MHz}$ RF pulses that are amplified with a $10 \mathrm{~W}$ broadband power amplifier. The RF pulses are sent to the high pass probe by a fast diode pin switch. The collected signed is amplified by a $50 \mathrm{~dB}$ low noise preamplifier and measured by a homodyne process br tho ijHFLI.

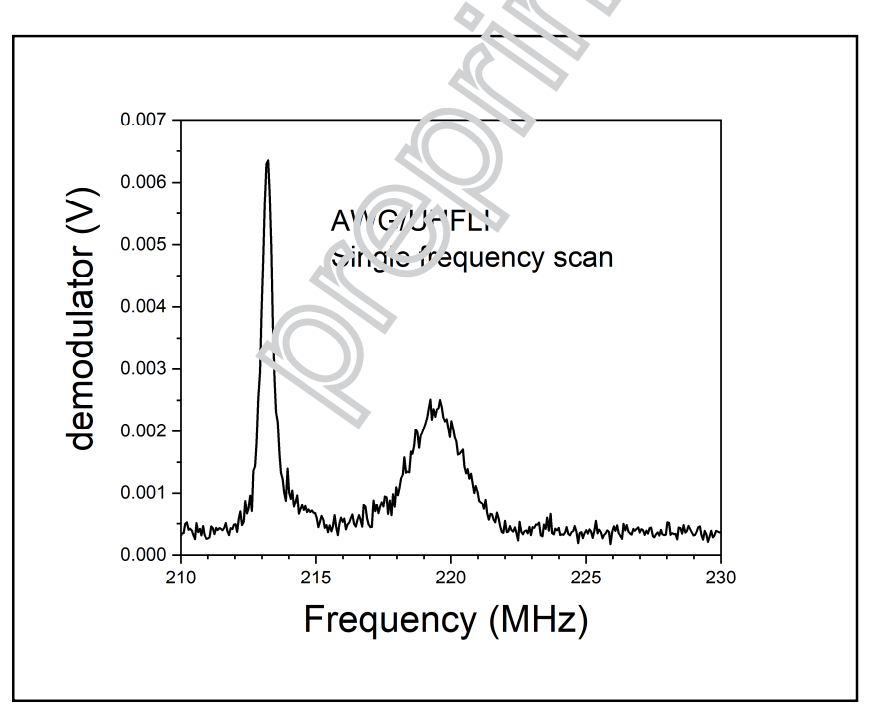

Figure 19. Single frequency scan recorded by the AWG/UHFLI based FNR set up for the same test sample as in figure 12. Spectra in figure 12 have been recorded after about 4000 data averaging for each frequency point. A similar averaging time would improve by a factor of 60 the signal to noise ratio. 